\title{
American Parisian options
}

\author{
Marc Chesney • Laurent Gauthier
}

Received: 4 July 2005 / Accepted: 22 March 2006 /

Published online: 11 August 2006

(C) Springer-Verlag 2006

\begin{abstract}
In this article, we describe the various sorts of American Parisian options and propose valuation formulae. Although there is no closed-form valuation for these products in the non-perpetual case, we have been able to reformulate their price as a function of the exercise frontier. In the perpetual case, closed-form solutions or approximations are obtained by relying on excursion theory. We derive the Laplace transform of the first instant Brownian motion reaches a positive level or, without interruption, spends a given amount of time below zero. We perform a detailed comparison of perpetual standard, barrier and Parisian options.
\end{abstract}

Keywords Parisian options · American options · Excursions

JEL Classification $\mathrm{G} 12 \cdot \mathrm{G} 13 \cdot \mathrm{C} 61 \cdot \mathrm{C} 65$

Mathematics Subject Classification (2000) $60 \mathrm{G} 40 \cdot 62 \mathrm{~L} 15 \cdot 60 \mathrm{~J} 65$

\section{Introduction}

In most articles concerning Parisian options, it is assumed that these options are of European type. Indeed, in [5] European Parisian options are defined

\footnotetext{
M. Chesney $(\varangle)$

University of Zurich, ISB, Plattenstrasse 14, Zurich 8032, Switzerland

e-mail: chesney@isb.unizh.ch

L. Gauthier

54 Greene Str., New York, NY 10013, USA

e-mail: lcgauthier@mac.com
} 
and priced (see also [6]). Bernard et al. [3] developed a new inverse Laplace transform method that is quick and appropriate to the pricing problem. Avellaneda and $\mathrm{Wu}$ [1] obtained a lattice scheme for calculating the price and sensitivities of such options. Costabile [7] provided a discrete time algorithm to evaluate European Parisian options (with flat or exponential barriers). Fujita and Miura [10] gave a new framework of barrier options to generalize Parisian options and "delayed barrier options".

However, in some papers, American Parisian options are also considered. Haber et al. [17] presented an approach to valuing European and American Parisian options using the numerical solution of a partial differential equation. Wilmott [21] also developed a PDE approach for these options. Grau [15] combined Monte Carlo simulations and PDE solvers (see also [16]) in order to price European and American Parisian options.

The objective of this paper is to develop a probabilistic approach for the pricing of American Parisian options. Even if the problem is complex, option prices can be obtained as a function of the exercise boundary. When maturity is infinite, the problem can, in most cases, be solved. Indeed, if the exercise boundary can be derived (as a constant) then the price can be obtained. In this paper, the analogies between the valuations of American (barrier) options and of American Parisian barrier options are explored. It is well known that American puts in the perpetual case (the so-called "Russian" options) can be priced with a closed-form formula. We show that this is also true for perpetual American Parisian puts. Possible approximations for long maturity American Parisian options can therefore be obtained.

We believe there are two important situations where closed-form expressions for American Parisian options can be used. First, there is real option analysis. One of the original ideas for this paper came from real option analysis in a duopoly where the optimal investment decision of one of the firms is represented with a Parisian option. This is along the lines of Gauthier [14] where the Parisian feature allows for the representation of a lag between an investment decision and its implementation. The other firm is for example more nimble and has no significant delay, and its investment decision can be represented as a simple barrier option (although with a higher entry cost). However, the smaller firm would be aware of the larger firm's strategy, and would therefore be in a position to preempt that larger firm's investment. As a result, the investment decision for the smaller firm can be represented as an American Parisian option. Obtaining a closed-form expression for the option's value allows for easier comparative statics. The second context in which a closed-form expression-albeit with simple assumptions concerning the dynamics of the underlying -is useful is the valuation of related options using Monte Carlo analysis. Indeed, one popular approach in the industry is the use of control variates, which requires the knowledge of a closed-form expression for some options in a limited context, and enforces the equality of the formula-based valuation and the result of the simulation. See for example [2] on the use of control variates to value lookback options in a stochastic volatility model, applying the known closed-form expression for the lookback option with a constant volatility. 
In this paper we focus purely on currency options, because we know that even the simplest options have non-trivial exercise frontiers in the American call case, and we shall be able to analyze correctly the behavior of the exercise frontier in all cases.

This article is organized as follows. After the introduction in Sect. 1, American Parisian options are described and classified in Sect. 2. Put-call parity results are also presented. In Sect. 3 the price of an American Parisian option is decomposed into two components, namely the price of the corresponding European Parisian option and the American premium. By relying on Chesney et al. [5], the first component is known. The second depends on the exercise boundary. For perpetual options, in most cases, the exercise boundary can be derived and prices of almost all types of American Parisian options can be obtained [as integrals of European Parisian (binary) option values for "out" options]. However, as shown in this section, in the case of perpetual American Parisian up-and-out call options the exercise boundary is not a constant. Therefore in this case, a closed-form solution cannot be obtained. In Sect. 4, by relying on [13] and [14], the problem is tackled from a different angle. The abovementioned analogies are used in the perpetual case, and closed-form expressions are derived. Concerning American Parisian down-and-out put (or similarly up-and-out call) options, simplifying assumptions are made. The approach used in this section is not directly connected with the decomposition results obtained in Sect. 3. A new result concerning the first instant when Brownian motion either hits a positive level or spends a certain time below zero is used in Sect. 4, and is shown in the appendix. Finally in Sect. 5, a brief conclusion is given and Sect. 6 contains the Appendix.

\section{American Parisian barrier options}

With traditional barrier options, everything is suddenly decided when the underlying price reaches the barrier: the option is either cancelled or activated. With a Parisian option, the underlying price has to spend a certain amount of time (called the "window" of the Parisian option) consecutively above or below the barrier. This makes it more difficult for a market player to manipulate the underlying price and force the cancellation or activation of the option.

There are quite a few combinations of features that define simple barrier options. We find the same variety in all the European Parisian barrier options, and a fortiori in all American Parisian barrier options. Essentially, there are eight types of barrier options corresponding to the combinations of up/down, in/out and call/put. By adding American/European and Parisian/standard (i.e. barrier) as additional combinations, we end up with a total of 32 products. For example, in the remainder of this paper, we denote by $C_{\mathrm{AP}}^{\mathrm{ui}}$ the price of an American Parisian up-and-in call. The price of a European Parisian down-andout call will be $C_{\mathrm{EP}}^{\mathrm{do}}$. While it is true that Parisian options are more complex than barrier options (there are closed-form solutions for barrier options, not for Parisian options), the value of a European Parisian option at time $t$ (before going 
through the barrier) still only depends on $t$ and on the underlying's value at that time-like a simple barrier option. The difference is that when the underlying is at the barrier, a simple barrier option's value is either zero or the value of a plain vanilla option, whereas in the case of a Parisian option, it is more complex.

\subsection{Using symmetry}

The first symmetry that comes to mind when analyzing options is the American put-call symmetry that applies to the case of foreign exchange options. The option to buy dollars versus Euros is an option to sell Euros versus dollars. Note that this symmetry applies to American as well as European options (see $[18,8])$ and also therefore to American Parisian and American barrier options (see also [5]). Formally, for European or American options, this is expressed as

$$
P\left(S_{t}, K, r, \delta\right)=S_{t} K C\left(1 / S_{t}, 1 / K, \delta, r\right),
$$

where $S_{t}$ is the exchange rate at a given time $t, K$ is the strike price in the corresponding unit and $r$ (resp. $\delta$ ) the domestic (resp. foreign) risk-free interest rate. $C$ and $P$ stand respectively for call and put (European or American). For American Parisian options, the put-call parity is expressed as

$$
\begin{aligned}
& P_{A P}^{d j}\left(S_{t}, K, L_{1}, D, r, \delta, T\right) \\
& \quad=S_{t} K C_{A P}^{u j}\left(1 / S_{t}, 1 / K, 1 / L_{1}, D, \delta, r, T\right), \quad \text { where } j \text { stands for } o \text { or } i, \\
& P_{A P}^{u j}\left(S_{t}, K, L_{1}, D, r, \delta, T\right) \\
& \quad=S_{t} K C_{A P}^{d j}\left(1 / S_{t}, 1 / K, 1 / L_{1}, D, \delta, r, T\right), \quad \text { where } j \text { stands for } o \text { or } i,
\end{aligned}
$$

where $L_{1}$ and $D$ are respectively the barrier and the window of the Parisian option (see the definitions in the next section). The case of the American (nonParisian) barrier option corresponds to $D=0$.

Standard European barrier options benefit from symmetry; for example, the sum of an up-and-in option and an up-and-out option with the same strike, barrier and maturity equals the plain vanilla option. However, this symmetry does not apply in the case of American options; the exercise frontier would have to be the same for the plain vanilla, the up-and-in and the up-and-out options. In the next subsection, we discuss in more detail why this is not the case.

\subsection{The American effect}

Thanks to the put-call symmetry, we can choose to consider either calls or puts in our analysis. We mostly concentrate on American options. Indeed, European option prices (plain vanilla, barrier and Parisian) are known. Furthermore, as already mentioned, barrier options correspond to a specific case of more general 
Parisian options, i.e. when the window is equal to zero. Therefore, in Sect. 3 we only consider American Parisian calls. Instead of 32 possible options, we focus on four cases corresponding to $C_{\mathrm{AP}}^{\mathrm{do}}, C_{\mathrm{AP}}^{\mathrm{uo}}, C_{\mathrm{AP}}^{\mathrm{di}}$ and $C_{\mathrm{AP}}^{\mathrm{ui}}$.

Let us first focus on the in/out features of barrier options (Parisian or standard). We know that in the case when there is no barrier, the American foreign exchange call has a non-trivial exercise frontier that is higher than the strike price and declines to the strike right before the option's maturity. If there is an "in" barrier, the option holder cannot do or decide anything before the option is activated; and once the option is activated, it does not have a barrier any more and is just a plain vanilla American call. The exercise frontier for an American "in" barrier option (whether Parisian or standard, up or down) is therefore the exercise frontier of the corresponding plain vanilla option, starting at the activation time. Hence, as shown in Sect. 3, the valuation of the option is easier in this case.

In the case of an "out" option, the situation is more complex than for an "in" option because of possible interactions between the incentives to exercise and the risk of losing the option. First, let us show that there is no in/out symmetry in the case of an American exercise. Let us consider a standard American up-and-out call where the barrier is higher than the strike price, but lower than the exercise frontier of the corresponding plain vanilla option. The exercise frontier for the up-and-out call is necessarily strictly below the barrier since at the barrier the option becomes worthless. Therefore, the exercise frontier of the up-and-out call is different from that of the plain vanilla option. Consequently, the value of the plain vanilla American option is different (strictly higher) from the sum of the "in" and "out" options.

For a call, the up-and-out case is more complex than the down-and-out. For a put, it is the contrary. When the barrier is higher than the strike (for the upand-out call), the risk of being knocked out interacts strongly with the exercise policy; it may become necessary to exercise the call, even sub-optimally, in order to avoid being knocked out. In the case of a down-and-out call, approaching the barrier does not trigger an early exercise of the call.

\section{Decomposition techniques for American Parisian options}

We assume that, under the risk-neutral probability $\mathbb{Q}$, the exchange rate on which our options are written solves the SDE

$$
\frac{\mathrm{d} S_{t}}{S_{t}}=(r-\delta) \mathrm{d} t+\sigma \mathrm{d} W_{t},
$$

where $S_{t}, r, \delta, \sigma\left(W_{t}, t \geq 0\right)$ are respectively the exchange rate at time $t$, the domestic and foreign risk-free rates, the volatility, and a $\mathbb{Q}$-Brownian motion. We assume that $\delta>0$. We have $S_{t}=x \mathrm{e}^{\sigma\left(\beta t+W_{t}\right)}$, or $S_{t}=x \mathrm{e}^{\sigma Z_{t}}$ where $S_{0}=x$ 
and $Z$ is a drifted Brownian motion, i.e. $\left(Z_{t}=\beta t+W_{t}, t \geq 0\right)$, with $\beta=\frac{r-\delta-\frac{\sigma^{2}}{2}}{\sigma}$. Let us now define the following functionals in terms of $S$ or in terms of $Z$ :

$$
\begin{aligned}
T_{L}(S)= & \inf \left\{t \geq 0: S_{t}=L\right\}=T_{l}(Z)=\inf \left\{t \geq 0: Z_{t}=l\right\}, \\
g_{t}^{L}(S)= & \sup \left\{u \in[0, t]: S_{u}=L\right\}=g_{t}^{l}(Z)=\sup \left\{u \in[0, t]: Z_{u}=l\right\}, \\
H_{L, D}^{+}(S)= & \inf \left\{t \geq 0:\left(t-g_{t}^{L}(S)\right) \geq D, S_{t} \geq L\right\} \\
= & H_{l, D}^{+}(Z)=\inf \left\{t \geq 0:\left(t-g_{t}^{l}(Z)\right) \geq D, Z_{t} \geq l\right\}, \\
H_{L, D}^{-}(S)= & \inf \left\{t \geq 0:\left(t-g_{t}^{L}(S)\right) \geq D, S_{t} \leq L\right\} \\
= & H_{l, D}^{-}(Z)=\inf \left\{t \geq 0:\left(t-g_{t}^{l}(Z)\right) \geq D, Z_{t} \leq l\right\}, \\
& \quad \text { with } l=\frac{\ln (L / x)}{\sigma} .
\end{aligned}
$$

They are, respectively, the first instant a process hits a given level, the last instant when the process was at a given level, and the two Parisian times: the first instant when the process spends consecutively more than $D$ units of time over or under a given level. Notice that $g_{t}^{h}(S)$ is not a stopping time. When this random time "happens", there is no way to know immediately that is has just happened. We write $H_{L, D}^{+/-}$for $H_{L, D}^{+/-}(S)$.

\subsection{The down-and-out Parisian call}

Definition 1 An American down-and-out Parisian call option entitles its owner to buy the underlying before the minimum of the maturity (which can be infinite) and the first instant when the underlying price spends, without interruption, more than $D$ units of time under a given level $L_{1}$ (the so-called Parisian time). If the investor decides to exercise at the stopping time $\tau$, the present value of the payoff of the option is $\mathrm{e}^{-r \tau}\left(S_{\tau}-K\right)^{+} \mathbb{I}_{\tau<H_{L_{1}, D}^{-}} \mathbb{I}_{\tau \leq T}$, where $T$ is the maturity and $K$ the strike price. We can consider that in most cases, we would have $L_{1} \leq K$.

Proposition 1 The price of an American Parisian down-and-out call can be decomposed as

$$
\begin{aligned}
C_{\mathrm{AP}}^{\mathrm{do}}(x, T)= & C_{\mathrm{EP}}^{\mathrm{do}}(x, T) \\
& +\delta x \int_{0}^{T} \exp \left(-\left(r+\frac{\beta^{2}}{2}\right) u\right) \mathbb{E}\left[\mathbb{I}_{Z_{u} \geqslant \bar{b}_{u}} \mathbb{I}_{u<H_{l_{1}, D}^{-}(Z)} \exp \left((\beta+\sigma) Z_{u}\right)\right] \mathrm{d} u \\
& -r K \int_{0}^{T} \exp \left(-\left(r+\frac{\beta^{2}}{2}\right) u\right) \mathbb{E}\left[\mathbb{I}_{Z_{u} \geqslant \bar{b}_{u}} \mathbb{I}_{u<H_{l_{1}, D}^{-}(Z)} \exp \left(\beta Z_{u}\right)\right] \mathrm{d} u,
\end{aligned}
$$


where $\beta=\frac{\left(r-\delta-\frac{\sigma^{2}}{2}\right)}{\sigma}, \quad \bar{b}_{u}=\frac{\ln \frac{\bar{S}_{u}}{x}}{\sigma}, \quad l_{1}=\frac{\ln \frac{L_{1}}{x}}{\sigma} \leq 0$ and where $\left\{\bar{S}_{u}, u \in\right.$ $[0, T]\}$ is the exercise boundary. The two expectations are defined by relying on the probability $\mathbb{P}$ under which $\left(Z_{t}=W_{t}+\beta t, t \geqslant 0\right)$, is a Brownian motion. This decomposition can also be written as

$$
C_{\mathrm{AP}}^{\mathrm{do}}(x, T)=C_{\mathrm{EP}}^{\mathrm{do}}(x, T)+\delta \int_{0}^{T}\left(C_{\mathrm{EP}}^{\mathrm{do}}\left(x, \bar{S}_{u}, u\right)+\left(\bar{S}_{u}-\frac{r}{\delta} K\right) \mathrm{BINC}_{\mathrm{EP}}^{\mathrm{do}}\left(x, \bar{S}_{u}, u\right)\right) \mathrm{d} u,
$$

where $C_{\mathrm{EP}}^{\mathrm{do}}\left(x, \bar{S}_{u}, u\right)$ is the price of the European Parisian down-and-out call option with maturity $u$, strike price $\bar{S}_{u}$, barrier $L_{1}$ and window $D$. $\mathrm{BINC}_{\mathrm{EP}}^{\mathrm{do}}\left(x, \bar{S}_{u}, u\right)$ is the price of a Parisian binary call which generates at maturity a payoff of one monetary unit if the underlying value is higher than the strike price and if the first instant-when the underlying price spends, without interruption, more than $D$ units of time under the level $L_{1}-$ is higher than the maturity $u$. Otherwise, the payoff is equal to zero.

Proof We first define the function

$$
f_{t}(x, v)=\exp (-r(v-t)) C_{\mathrm{AP}}^{\mathrm{do}}(x, T-v) .
$$

We plan to write the price of the option as a function of the underlying's price, and to apply stochastic calculus techniques. By applying Itô's formula, we obtain

$$
\begin{aligned}
f_{t}\left(S_{T}, T\right)= & \exp (-r(T-t)) C_{\mathrm{AP}}^{\mathrm{do}}\left(S_{T}, 0\right) \\
= & C_{\mathrm{AP}}^{\mathrm{do}}\left(S_{t}, T-t\right)+\int_{t}^{T} \mathcal{L}\left(\exp (-r(u-t)) C_{\mathrm{AP}}^{\mathrm{do}}\left(S_{u}, T-u\right)\right) \mathrm{d} u \\
& +\int_{t}^{T} \exp (-r(u-t)) \frac{\partial C_{\mathrm{AP}}^{\mathrm{do}}}{\partial x}\left(S_{u}, T-u\right) \sigma S_{u} \mathrm{~d} W_{u},
\end{aligned}
$$

where the differential operator $\mathcal{L}$, the infinitesimal generator of the diffusion $S$, is defined by

$$
\mathcal{L}=\frac{1}{2} \sigma^{2} S^{2} \frac{\partial^{2}}{\partial x^{2}}+(r-\delta) S \frac{\partial}{\partial x}+\frac{\partial}{\partial u} .
$$

The second derivative is understood in a weak sense, as the operator is applied to functions that are not twice differentiable, but are differences of convex 
functions. Taking expectations and recognizing the third term to be a martingale, we obtain

$$
\begin{aligned}
\mathbb{E} & {\left[\exp (-r(T-t)) C_{\mathrm{AP}}^{\mathrm{do}}\left(S_{T}, 0\right) \mid \mathcal{F}_{t}\right] } \\
= & C_{\mathrm{AP}}^{\mathrm{do}}\left(S_{t}, T-t\right)+\mathbb{E}\left[\int_{t}^{T} \mathcal{L}\left(\exp (-r(u-t)) C_{\mathrm{AP}}^{\mathrm{do}}\left(S_{u}, T-u\right)\right) \mathrm{d} u \mid \mathcal{F}_{t}\right] .
\end{aligned}
$$

To clarify our notations, we have written in the above equation $\mathcal{L}(f(u))$ instead of $\mathcal{L} f(u)$. We keep the same notation in the remainder of the paper. Let us now assume that $t=0$.

We know the value of the option at certain times or at certain levels, and exploit that through the infinitesimal generator. When the option is neither cancelled or exercised, that is in the so-called continuation region, we have

$$
\mathcal{L}\left(\exp (-r(u-t)) C_{\mathrm{AP}}^{\mathrm{do}}\left(S_{u}, T-u\right)\right)=0 .
$$

When the option is exercised, its value is $S-K, S$ being the underlying value at that time. In the case of the down-and-out option, we know that $S_{H_{L_{1}, D}^{-}} \leq K$, whenever the Parisian time is triggered, so there is no interest in exercising the option. So $\mathcal{L}\left(\exp (-r u) C_{\mathrm{AP}}^{\mathrm{do}}\left(S_{u}, T-u\right)\right)$ is non-zero only on $\left\{S_{u} \geq \bar{S}_{u}\right\} \cap$ $\left\{u<H_{L_{1}, D}^{-}\right\}$where $\left(\bar{S}_{u}, u \geqslant 0\right)$ is the exercise boundary for the call, i.e.

$$
\bar{S}_{u}=\inf \left\{y \geqslant 0: y-K=C_{\mathrm{AP}}^{\mathrm{do}}(y, T-u)\right\} .
$$

So in these cases, we have $C_{\mathrm{AP}}^{\mathrm{do}}\left(S_{u}, T-u\right)=S_{u}-K$. Using the definition of the generator and (3.4), we see that the call price is given by

$$
\begin{aligned}
C_{\mathrm{AP}}^{\mathrm{do}}(x, T)= & \mathbb{E}\left[\exp (-r T)\left(S_{T}-K\right)^{+} \mathbb{I}_{H_{L_{1}, D}^{-}>T}\right] \\
& -\mathbb{E}\left[\int_{0}^{T} \mathrm{e}^{-r u}\left(r K-\delta S_{u}\right) \mathbb{I}_{S_{u} \geqslant \bar{S}_{u}} \mathbb{I}_{u<H_{L_{1}, D}^{-}} \mathrm{d} u\right] .
\end{aligned}
$$

The first term on the right-hand side is the price $C_{\mathrm{EP}}^{\mathrm{do}}$ of a European Parisian down-and-out call option. The valuation of such options is presented in [5]. Equation (3.5) can be rewritten as 


$$
\begin{aligned}
C_{\mathrm{AP}}^{\mathrm{do}}(x, T)= & C_{\mathrm{EP}}^{\mathrm{do}}(x, T)+\delta \int_{0}^{T} \exp (-r u) \mathbb{E}\left[S_{u} \mathbb{I}_{\beta u+W_{u} \geqslant \frac{\ln \left(\bar{S}_{u} / S_{0}\right)}{\sigma}} \mathbb{I}_{u<H_{L_{1}, D}^{-}}\right] \mathrm{d} u \\
& -r K \int_{0}^{T} \exp (-r u) \mathbb{E}\left[\mathbb{I}_{\beta u+W_{u} \geqslant \frac{\ln \left(\bar{S}_{u} / S_{0}\right)}{\sigma}} \mathbb{I}_{u<H_{L_{1}, D}^{-}}\right] \mathrm{d} u .
\end{aligned}
$$

By introducing a new probability $\mathbb{P}$ under which $\left(Z_{t}=W_{t}+\beta t, t \geqslant 0\right)$, is a Brownian motion and by relying on Girsanov's theorem, the first decomposition of Proposition 1 is obtained. The decomposition technique used in order to derive this result is similar to the approach followed by Gao et al. [11] in the non-Parisian case. Furthermore, (3.5) yields

$$
C_{\mathrm{AP}}^{\mathrm{do}}(x, T)=C_{\mathrm{EP}}^{\mathrm{do}}(x, T)+\delta \int_{0}^{T} E\left[\mathrm{e}^{-r u}\left(S_{u}-\frac{r}{\delta} K\right) \mathbb{I}_{S_{u} \geqslant \bar{S}_{u}} \mathbb{I}_{H_{L_{1}, D}^{-}>u}\right] \mathrm{d} u .
$$

The expectation on the right-hand side can be written as

$$
\begin{aligned}
& E\left[\mathrm{e}^{-r u}\left(S_{u}-\frac{r}{\delta} K\right) \mathbb{I}_{S_{u} \geqslant \bar{S}_{u}} \mathbb{I}_{H_{L_{1}, D}^{-}>u}\right] \\
& \quad=\mathrm{e}^{-r u} E\left[\left(S_{u}-\bar{S}_{u}\right) \mathbb{I}_{S_{u} \geqslant \bar{S}_{u}} \mathbb{I}_{H_{L_{1}, D}^{-}>u}\right]+\left(\bar{S}_{u}-\frac{r}{\delta} K\right) \mathrm{e}^{-r u} P\left(S_{u} \geqslant \bar{S}_{u}, H_{L_{1}, D}^{-}>u\right) .
\end{aligned}
$$

By definition, the first term on the right-hand side of (3.8) is, at time zero, the price $C_{\mathrm{EP}}^{\mathrm{do}}\left(x, \bar{S}_{u}, u\right)$ of the European Parisian down-and-out call option with maturity $u$, strike price $\bar{S}_{u}$, barrier $L_{1}$ and window $D$. The second term is the value of $\left(\bar{S}_{u}-\frac{r}{\delta} K\right)$ (this term is positive because $\left.\bar{S}_{u} \geqslant \sup \left(\frac{r}{\delta} K, K\right) \forall u\right)$ Parisian binary calls defined in Proposition 1 . The value of such an option, denoted BINC $_{\mathrm{EP}}^{\mathrm{do}}\left(x, \bar{S}_{u}, u\right)$, is therefore equal to the opposite of the derivative of the European Parisian option with respect to the strike (see [9]). Hence the second decomposition of Proposition 1 is obtained, with

$$
\operatorname{BINC}_{\mathrm{EP}}^{\mathrm{do}}\left(x, \bar{S}_{u}, u\right)=-\frac{\mathrm{d} C_{\mathrm{EP}}^{\mathrm{do}}\left(x, \bar{S}_{u}, u\right)}{\mathrm{d} \bar{S}_{u}}
$$

It is possible to compute the Laplace transform of the binary call (see [5] in the case of the Laplace transform of the Delta of the European Parisian option).

\section{The perpetual case}

In the perpetual case the following proposition is obtained. 
Proposition 2 The price of a perpetual American Parisian down-and-out call is given by

$$
\begin{aligned}
C_{\mathrm{AP}}^{\mathrm{do}}(x)= & \delta \int_{0}^{+\infty}\left(C_{\mathrm{EP}}^{\mathrm{do}}\left(x, L_{C}, u\right)+\left(L_{C}-\frac{r}{\delta} K\right) \mathrm{BINC}_{\mathrm{EP}}^{\mathrm{do}}\left(x, L_{C}, u\right)\right) \mathrm{d} u \\
= & \left(1-\frac{\Phi\left(-\sqrt{\left(2 r+\beta^{2}\right) D}\right)}{\Phi\left(\sqrt{\left(2 r+\beta^{2}\right) D}\right)} \exp \left(2 l_{1} \sqrt{2 r+\beta^{2}}\right)\right) \\
& \times \frac{1}{\sigma \sqrt{2 r+\beta^{2}}}\left(\frac{x}{L_{C}}\right)^{\theta}\left(\frac{\delta L_{C}}{\theta-1}-\frac{r}{\theta} K\right) \quad \text { with } \theta=\frac{-\beta+\sqrt{2 r+\beta^{2}}}{\sigma},
\end{aligned}
$$

where the exercise boundary $L_{C}$ is obtained by relying on the equation

$$
L_{C}-K=\left(1-\frac{\Phi\left(-\sqrt{\left(2 r+\beta^{2}\right) D}\right)}{\Phi\left(\sqrt{\left(2 r+\beta^{2}\right) D}\right)}\left(\frac{L_{1}}{L_{C}}\right)^{2 \sqrt{2 r+\beta^{2}} / \sigma}\right) \frac{1}{\sigma \sqrt{2 r+\beta^{2}}}\left(\frac{\delta L_{C}}{\theta-1}-\frac{r}{\theta} K\right)
$$

and where

$$
\Phi(z)=\int_{0}^{+\infty} x \exp \left(z x-\frac{x^{2}}{2}\right) \mathrm{d} x=1+z \sqrt{2 \pi} \exp \left(\frac{z^{2}}{2}\right) N(z) .
$$

Proof Let us first consider the exercise policy in the perpetual case. As shown by Mordecki [19] for standard American options (in the case of diffusions with jumps), one can consider only stopping times which are hitting times, i.e. stopping times of the form

$$
T_{L}=\inf \left\{t \geqslant 0: S_{t} \geqslant L\right\}
$$

In other words the exercise boundary is flat. Let us denote it by $L_{C}$. The argument in [19] can easily be extended to standard American down-and-out barrier options and to American Parisian down-and-out call options. Hence, by taking $T \rightarrow \infty$ in Proposition 1, the first decomposition of Proposition 2 is obtained. $C_{\mathrm{AP}}^{\mathrm{do}}(x)$ stands for $C_{\mathrm{AP}}^{\mathrm{do}}(x,+\infty)$. Indeed, the price of the European Parisian option disappears because

$$
\lim _{T \rightarrow \infty} C_{\mathrm{EP}}^{\mathrm{do}}(x, T) \leqslant \lim _{T \rightarrow \infty} C_{E}(x, T)=0, \quad \forall \delta>0,
$$

where $C_{E}(x, T)$ is the price of the standard European currency option, given by Garman and Kohlhagen [12]. The exercise boundary $L_{C}$ is then obtained as

$$
L_{C}=\inf \left\{y \geqslant 0: C_{\mathrm{AP}}^{\mathrm{do}}(y)=y-K\right\} .
$$


This means that in the exercise region, the value of the option is equal to its intrinsic value. By assuming that $x=L_{C}$ in the first decomposition of Proposition 2 , we obtain

$$
L_{C}-K=\delta \int_{0}^{+\infty}\left(C_{\mathrm{EP}}^{\mathrm{do}}\left(L_{C}, L_{C}, u\right)+\left(L_{C}-\frac{r}{\delta} K\right) \mathrm{BINC}_{\mathrm{EP}}^{\mathrm{do}}\left(L_{C}, L_{C}, u\right)\right) \mathrm{d} u .
$$

As shown in Appendix A, these results can be further simplified and the full proposition is obtained.

\section{The case of an already started excursion}

In this case, we assume that, at time zero, the excursion has already started (see [5]). The impact of such a situation on the value of the option is in fact very simple; either the underlying price hits the barrier back again quickly enough in the remaining time, or it does not. If it does not, the option is cancelled at the Parisisan time. So the option's value will be the value starting from the barrier level weighted by the probability that the underlying price hits it before it is too late.

More specifically, let us assume that the excursion started $u$ units of time ago, where $u<D$. In this context, the Parisian option price has now three arguments: the initial value $x$ of the underlying, time $T$ to maturity and $u$. Then, if the price does not go back up to $L_{1}$ before $D-u$ units of time, the option will be cancelled. At time zero, the underlying price $x$ has to be under $L_{1}$ since the excursion has started. So we can consider $T_{L_{1}}$, the hitting time of the barrier. If the price hits the barrier before $D-u$ units of time, we revert to the previous case again and the price of the option is known. It is $C_{\mathrm{AP}}^{\mathrm{do}}\left(L_{1}, T-T_{L_{1}}, 0\right)$. So,

$$
\begin{aligned}
C_{\mathrm{AP}}^{\mathrm{do}}(x, T, u) & =\mathbb{E}_{x}\left[\mathbb{I}_{T_{L_{1}} \leq D-u} \mathrm{e}^{-r T_{L_{1}}} C_{\mathrm{AP}}^{\mathrm{do}}\left(L_{1}, T-T_{L_{1}}, 0\right)\right] \\
& =\int_{0}^{D-u} \mathbb{P}_{x}\left(T_{L_{1}} \in \mathrm{d} s\right) \mathrm{e}^{-r s} C_{\mathrm{AP}}^{\mathrm{do}}\left(L_{1}, T-s, 0\right)
\end{aligned}
$$

and

$$
C_{\mathrm{AP}}^{\mathrm{do}}(x,+\infty, u)=\mathbb{E}_{x}\left[\mathbb{I}_{T_{L_{1}} \leq D-u} \mathrm{e}^{-r T_{L_{1}}}\right] C_{\mathrm{AP}}^{\mathrm{do}}\left(L_{1}\right),
$$

where $C_{\mathrm{AP}}^{\mathrm{do}}\left(L_{1}, T-s, 0\right)=C_{\mathrm{AP}}^{\mathrm{do}}\left(L_{1}, T-s\right)$ is given by Proposition 1 . In addition, the term $\mathbb{E}_{x}\left[\mathbb{I}_{T_{L_{1}} \leq D-u} \mathrm{e}^{-r T_{L_{1}}}\right]$ is known, since it depends solely on the law of $T_{L_{1}}(S)$. We have

$$
\mathbb{E}_{x}\left[\mathbb{I}_{T_{L_{1}} \leq D-u} \mathrm{e}^{-r T_{L_{1}}}\right]=\mathbb{E}\left[\mathbb{I}_{T_{l_{1}}(Z) \leq D-u} \mathrm{e}^{-r T_{l_{1}}(Z)}\right]
$$


for $Z_{t}=W_{t}+\beta t, t \geqslant 0$, and when $x>L_{1}$ (i.e. $l_{1}<0$, where $l_{1}$ is defined in Proposition 1). Using [4, p. 223], we obtain

$$
\mathbb{E}_{x}\left[\mathbb{I}_{T_{L_{1}} \leq D-u} \mathrm{e}^{-r T_{L_{1}}}\right]=\int_{0}^{D-u} \frac{\left|l_{1}\right|}{\sqrt{2 \pi} s^{\frac{3}{2}}} \mathrm{e}^{-r s-\frac{1}{2 s}\left(l_{1}-\beta s\right)^{2}} \mathrm{~d} s .
$$

\subsection{The up-and-out Parisian call}

Definition 2 An American up-and-out Parisian call option entitles its owner to buy the underlying before the minimum of the maturity and the first instant when the underlying price spends, without interruption, more than D units of time over a given level $L_{1}$ (the so-called Parisian time). If the investor decides to exercise at the stopping time $\tau$, the present value of the payoff of the option is $\mathrm{e}^{-r \tau}\left(S_{\tau}-K\right)^{+} \mathbb{I}_{\tau<H_{L_{1}, D}^{+}} \mathbb{I}_{\tau \leq T}$, where $T$ is the maturity and $K$ the strike price.

Proposition 3 The price of an American Parisian up-and-out call option can be decomposed as

$$
\begin{aligned}
& C_{\mathrm{AP}}^{\mathrm{uo}}(x, T)=C_{\mathrm{EP}}^{\mathrm{uo}}(x, T)+\int_{0}^{T} \exp \left(-\left(r+\frac{\beta^{2}}{2}\right) u\right) \\
& \times \mathbb{E}\left[\mathbb{I}_{Z_{u} \geq \bar{b}_{u}} \mathbb{I}_{u<H_{l_{1}, D}^{+}(Z)}\left(\delta x \exp \left(\sigma Z_{u}\right)-r K\right) \mathrm{e}^{\beta Z_{u}}\right] \mathrm{d} u,
\end{aligned}
$$

where $\beta, \bar{b}_{u}$ and $l_{1}$ are defined in Proposition 1 and where $\left\{\bar{S}_{u}, u \in[0, T]\right\}$ is the exercise boundary. The expectation is defined by relying on the probability $\mathbb{P}$ under which $Z_{t}=W_{t}+\beta t, t \geqslant 0$, is a Brownian motion. This decomposition can also be written as

$$
C_{\mathrm{AP}}^{\mathrm{uo}}(x, T)=C_{\mathrm{EP}}^{\mathrm{uo}}(x, T)+\delta \int_{0}^{T}\left(C_{\mathrm{EP}}^{\mathrm{uo}}\left(x, \bar{S}_{u}, u\right)+\left(\bar{S}_{u}-\frac{r}{\delta} K\right) \operatorname{BINC}_{\mathrm{EP}}^{\mathrm{uo}}\left(x, \bar{S}_{u}, u\right)\right) \mathrm{d} u .
$$

Proof By relying on the argument used for the down-and-out call, this proposition can be straightforwardly obtained. Indeed, we know that $\mathcal{L}\left(\exp (-r u) C_{\mathrm{AP}}^{\mathrm{uo}}\left(S_{u}, T-u\right)\right)$ is non-zero only on $\left\{S_{u} \geqslant \bar{S}_{u}\right\}$ and $\left\{u<H_{L_{1}, D}^{+}\right\}$; so $\mathcal{L}\left(\exp (-r u) C_{\mathrm{AP}}^{\mathrm{uo}}\left(S_{u}, T-u\right)\right)=\mathrm{e}^{-r u}\left(r K-\delta S_{u}\right) \mathbb{I}_{\left\{S_{u} \geq \bar{S}_{u}\right\} \cap\left\{u<H_{L_{1}, D}^{+}\right\}}$and the result is obtained.

It is worthwhile to look further at this exercise boundary. When the exercise boundary of the standard American call is higher than the barrier $L_{1}, \bar{S}$ is defined only when the underlying value $S_{u}$ is higher than $L_{1}$. In these cases 
$\bar{S}_{u}$ depends not only on time $u$, but also on $g_{u}^{L_{1}}(S)$, the last instant when the process $S$ was at level $L_{1}$. As long as $S$ remains above $L_{1}$, the exercise boundary is defined on the interval $\left[g_{u}^{L_{1}}(S), g_{u}^{L_{1}}(S)+D\right]$. This exercise boundary is a decreasing function of time and reaches $L_{1}$ at time $g_{u}^{L_{1}}(S)+D$. Indeed, if the process remains above the barrier $L_{1}$, the option should be exercised before time $g_{u}^{L_{1}}(S)+D$, otherwise it will be knocked out.

If the underlying value does not reach the exercise boundary before time $g_{u}^{L_{1}}(S)+D$, this implies that the underlying has reached $L_{1}$ from above before this time. The exercise boundary will be activated the next time that the underlying reaches the barrier $L_{1}$ from below. When the exercise boundary of the standard American call is smaller than the barrier $L_{1}$, then $\bar{S}$ is this exercise boundary.

This description of the exercise is also true in the perpetual case. When the exercise boundary of the standard American call is higher than the barrier $L_{1}$, the exercise boundary $\bar{S}$ is not a constant. Otherwise, $\bar{S}$ is equal to this exercise boundary. It therefore does not appear to be directly possible to calculate the value of the American Parisian up-and-out call option in the perpetual case with a closed-form expression. The main issue is that in some cases, the exercise boundary is not a constant. This is because even in the perpetual case, the window of the Parisian option generates a finite maturity. Indeed, the option can be knocked out if the Parisian time is reached. We shall see in Sect. 4 (in the equivalent case of the down-and-out put) how we can address this problem, by simplifying our assumptions.

\subsection{The "in" Parisian call}

As previously mentioned, with an "in" barrier, the option holder cannot do or decide anything before the option is activated; once the option is activated, it no longer has a barrier and is just a plain vanilla call. Therefore

$$
\begin{aligned}
C_{\mathrm{AP}}^{\mathrm{di}}(x, T) & =\mathbb{E}\left[\exp \left(-r H_{L_{1}, D}^{-}\right) \mathbb{I}_{H_{L_{1}, D}^{-} \leqslant T} C_{\mathrm{A}}\left(S_{H_{L_{1}, D}^{-}}, T-H_{L_{1}, D}^{-}\right)\right] \\
& =\int_{0}^{T} \exp (-r u) C_{\mathrm{A}}\left(S_{u}, T-u\right) Q\left(H_{L_{1}, D}^{-} \in \mathrm{d} u\right) .
\end{aligned}
$$

In the perpetual case, this gives

$$
C_{\mathrm{AP}}^{\mathrm{di}}(x)=\mathbb{E}\left[\exp \left(-r H_{L_{1}, D}^{-}\right)\right] \mathbb{E}\left[C_{\mathrm{A}}\left(S_{H_{L_{1}, D}^{-}}\right)\right] .
$$

Indeed, the random variables $H_{l_{1}, D}^{-}(Z)$ and $Z_{H_{l_{1}, D}^{-}(Z)}$ are independent, and the law of $Z_{H_{l_{1}, D}^{-}(Z)}$ and the Laplace transform of $H_{L_{1}, D}^{-}$are given by (see [5]) 


$$
\begin{aligned}
& P\left(Z_{H_{l_{1}, D}^{-}(Z)} \in \mathrm{d} y\right)=\frac{\mathrm{d} y}{D} \mathbb{I}_{y<l_{1}}\left(l_{1}-y\right) \exp \left(-\frac{\left(y-l_{1}\right)^{2}}{2 D}\right), \\
& \mathbb{E}\left[\left(\exp \left(-\frac{\lambda^{2}}{2} H_{L_{1}, D}^{-}\right)\right]=\frac{\exp \left(l_{1} \lambda\right)}{\Phi(\lambda \sqrt{D})},\right.
\end{aligned}
$$

where the function $\Phi$ is given in Proposition 2.

The value of the standard American option is also known; it is $C_{\mathrm{A}}(x)=$ $\left(L_{C}-K\right)\left(\frac{x}{L_{C}}\right)^{\theta}$, where $\theta$ is given in Proposition 2. The exercise boundary of the call is the constant $L_{C}=\frac{\theta}{\theta-1} K$.

When the American Parisian call is activated, its exercise boundary is also $L_{C}$. Therefore when the expectation is defined by relying on the probability $\mathbb{P}$ under which $\left(Z_{t}=W_{t}+\beta t, t \geqslant 0\right)$, is a Brownian motion, then

$$
\begin{aligned}
C_{\mathrm{AP}}^{\mathrm{di}}(x) & =\mathbb{E}\left[\exp \left(-\left(r+\frac{\beta^{2}}{2}\right) H_{L_{1}, D}^{-}\right)\right] \mathbb{E}\left[\mathrm{e}^{\left.\beta Z_{H_{l_{1}, D}^{-}(Z)} C_{\mathrm{A}}\left(x \mathrm{e}^{\sigma Z_{H_{l_{1}, D}^{-}(Z)}}\right)\right]}\right. \\
& =x^{\theta} \frac{\exp \left(l_{1} \sqrt{2 r+\beta^{2}}\right)}{\Phi\left(\sqrt{\left(2 r+\beta^{2}\right) D}\right)} \frac{\left(L_{C}-K\right)}{L_{C}^{\theta}} \int_{-\infty}^{l_{1}} \mathrm{e}^{(\beta+\sigma \theta) y} P\left(Z_{H_{l_{1}, D}^{-}(Z)} \in \mathrm{d} y\right) .
\end{aligned}
$$

For American Parisian barrier calls, i.e. when the window $D$ is zero,

$$
C_{\mathrm{AP}}^{\mathrm{di}}(x)=C_{\mathrm{A}}\left(L_{1}\right) \mathbb{E}\left[\exp \left(-r T_{L_{1}}\right)\right]
$$

Along the same lines for the American Parisian up-and-in call, we obtain the formula

$$
C_{\mathrm{AP}}^{\mathrm{ui}}(x, T)=\int_{0}^{T} \exp (-r u) C_{\mathrm{A}}\left(S_{u}, T-u\right) Q\left(H_{L_{1}, D}^{+} \in \mathrm{d} u\right) .
$$

In the perpetual case,

$$
C_{\mathrm{AP}}^{\mathrm{ui}}(x)=\mathbb{E}\left[\exp \left(-\left(r+\frac{\beta^{2}}{2}\right) H_{L_{1}, D}^{+}\right)\right] \mathbb{E}\left[\mathrm{e}^{\beta Z_{H_{l_{1}, D}^{+}}(Z)} C_{\mathrm{A}}\left(x \mathrm{e}^{\sigma Z_{H_{l_{1}, D}^{+}}(Z)}\right)\right]
$$

By symmetry, the Laplace transform of the Parisian time $H_{L_{1}, D}^{+}$is known. Hence,

$$
\begin{aligned}
C_{\mathrm{AP}}^{\mathrm{ui}}(x)= & \frac{\exp \left(-l_{1} \sqrt{2 r+\beta^{2}}\right)}{\Phi\left(\sqrt{\left(2 r+\beta^{2}\right) D}\right)}\left(\frac{x^{\theta}\left(L_{C}-K\right)}{L_{C}^{\theta}} \int_{l_{1}}^{l_{C}} \mathrm{e}^{(\beta+\sigma \theta) y} P\left(Z_{H_{l_{1}, D}^{+}(Z)} \in \mathrm{d} y\right)\right. \\
& \left.+\int_{l_{C}}^{+\infty} \mathrm{e}^{\beta y}\left(x \mathrm{e}^{\sigma y}-K\right) P\left(Z_{H_{l_{1}, D}^{+}(Z)} \in \mathrm{d} y\right)\right)
\end{aligned}
$$


with $L_{C}>L_{1}$, and where $l_{C}=\ln \left(L_{C} / x\right) / \sigma$ and

$$
P\left(Z_{H_{l_{1}, D}^{+}(Z)} \in \mathrm{d} y\right)=\frac{\mathrm{d} y}{D} \mathbb{I}_{y>l_{1}}\left(y-l_{1}\right) \exp \left(-\frac{\left(y-l_{1}\right)^{2}}{2 D}\right) .
$$

Indeed, if $Z_{H_{l_{1}, D}^{+}(Z)}>l_{C}$, i.e., if $S_{H_{L_{1}, D}^{+}}>L_{C}$, then the American Parisian upand-in call should be exercised at the first time it is knocked in, i.e. at time $H_{L_{1}, D^{*}}^{+}$

\section{Analogies between American (barrier) and American Parisian barrier options}

In the non-perpetual case, it is unfortunately impossible to derive closed-form formulae. The perpetual case is therefore the only way we can perform some qualitative analysis of American Parisian options. Let us now tackle the problem from a different angle than in the last section and consider the perpetual case directly. Basically, we shall manipulate Laplace transforms of stopping times, combined with the position of the Brownian motion at these times. This approach is not directly connected with Propositions 1 and 2 and is mainly based on the fourth chapter of [13].

We carry out our analysis by starting with the simplest case, namely nonParisian, non-barrier options. The put case is now considered (the results can be directly used in the case of a non-dividend paying stock, $\delta=0$ ). We show how the value of an American put can be derived with a purely probabilistic approach. Then we deal with American non-Parisian perpetual barrier puts, and finally treat the Parisian case. We focus only on up-and-out and down-andout puts. Indeed, as shown in Sect. 3, "in" American option prices can be quite easily derived. The new approach used in this section generates an approximation for the option price in the difficult down-and-out put case (or similarly in the up-and-in call case studied in Sect. 3).

\subsection{The American perpetual put and the American perpetual barrier put}

The value of a perpetual American put is known. However, we state it here to illustrate the approach - where expected discounted payoffs are computed for a given exercise boundary - which we follow with more complex options later on. The well-known result is

Proposition 4 The value of the standard American perpetual put is the supremum over all possible optimal levels $L$ of the expected discounted cash flows under the risk-neutral probability, i.e. $P_{\mathrm{A}}(x)=\sup _{L} \mathbb{E}\left[\mathrm{e}^{-r T_{L}}(K-L)\right]$. This value can be calculated and we have $P_{\mathrm{A}}(x)=\left(K-L_{P}\right)\left(\frac{L_{P}}{x}\right)^{\xi}$, with $\xi=\frac{\beta+\sqrt{2 r+\beta^{2}}}{\sigma}$, and the exercise boundary of the put is $L_{P}=\frac{\xi}{\xi+1} K$. 
Proof Let us first calculate the value of the option as a function of the exercise frontier. We note that $T_{L}(S)=T_{l}(Z)$, where $Z$ is the drifted Brownian motion starting from zero, and where $l=\frac{1}{\sigma} \ln \frac{L}{x}$ and $\beta=\frac{1}{\sigma}\left(r-\delta-\frac{\sigma^{2}}{2}\right)$. In consequence, we can apply Girsanov's theorem at that stopping time and we have

$$
\mathbb{E}_{x}\left[\mathrm{e}^{-r T_{L}}(K-L)\right]=(K-L) \mathbb{E}_{0}\left[\mathrm{e}^{\beta l-\left(r+\frac{\beta^{2}}{2}\right) T_{l}(Z)}\right]=(K-L)\left(\frac{L}{x}\right)^{\xi}
$$

To determine the optimal $L$, we just solve $\frac{\partial}{\partial L}\left((K-L)\left(\frac{L}{x}\right)^{\xi}\right)=0$. We find that $L_{P}=\frac{\xi}{\xi+1} K$ solves this equation.

\section{Up-and-out and down-and-out}

The up-and-out case is the most complex case from a formulation perspective. An American down-and-out put is relatively trivial. If the barrier is lower than the optimal exercise level in the non-barrier case, then the barrier does not change anything, and the exercise should be at $L_{P}=\frac{\xi}{\xi+1} K$. If on the contrary the barrier is higher, then the option should be exercised right before the barrier. With an up-and-out, the dynamics will be different. The risk of losing the option if $S$ goes up will make it optimal to exercise the option at a higher threshold than $\frac{\xi}{\xi+1} K$. The following result is obtained.

Proposition 5 The value of the perpetual up-and-out American put with the barrier at $L_{1}$ verifies

$$
P_{\mathrm{A}}^{\mathrm{uo}}(x)=\sup _{L} \mathbb{E}_{x}\left[\mathrm{e}^{-r T_{L}}(K-L) \mathbb{I}_{T_{L}<T_{L_{1}}}\right]=\left(K-L_{P}\right) \frac{\left(\frac{L_{1}}{x}\right)^{\gamma}-\left(\frac{L_{1}}{x}\right)^{-\gamma}}{\left(\frac{L_{1}}{L_{P}}\right)^{\gamma}-\left(\frac{L_{1}}{L_{P}}\right)^{-\gamma}}\left(\frac{L_{P}}{x}\right)^{\frac{\beta}{\sigma}},
$$

with $\gamma=\frac{1}{\sigma} \sqrt{2 \rho}, \rho=r+\frac{\beta^{2}}{2}$, and where $L_{P}$ solves

$$
\frac{\partial}{\partial L}\left[(K-L) \frac{\left(\frac{L_{1}}{x}\right)^{\gamma}-\left(\frac{L_{1}}{x}\right)^{-\gamma}}{\left(\frac{L_{1}}{L}\right)^{\gamma}-\left(\frac{L_{1}}{L}\right)^{-\gamma}}\left(\frac{L}{x}\right)^{\frac{\beta}{\sigma}}\right]=0
$$

Proof The argument relies on Brownian hitting times. We follow the same approach as in the previous proof. Note that we always assume $L_{1} \geq x \geq L$. We write, applying Girsanov's theorem at $T_{L}$,

$$
\mathbb{E}_{x}\left[\mathrm{e}^{-r T_{L}}(K-L) \mathbb{I}_{T_{L}<T_{L_{1}}}\right]=(K-L) \mathbb{E}_{0}\left[\mathrm{e}^{l \beta-\rho T_{l}} \mathbb{I}_{T_{l}<T_{l_{1}}}\right],
$$


where $l_{1}=\frac{1}{\sigma} \ln \frac{L_{1}}{x}$. Now, it is well known (see [4, p. 163] for example) that

$$
\mathbb{E}_{a}\left[\mathrm{e}^{-r T_{0}} \mathbb{I}_{T_{0} \leq T_{y}}\right]=\frac{\sinh ((y-a) \sqrt{2 r})}{\sinh (y \sqrt{2 r})}
$$

for a Brownian motion. Applying this result, we obtain

$$
\mathbb{E}_{\mathcal{X}}\left[\mathrm{e}^{-r T_{L}} \mathbb{I}_{T_{L}<T_{L_{1}}}\right]=\frac{\left(\frac{L_{1}}{x}\right)^{\gamma}-\left(\frac{L_{1}}{x}\right)^{-\gamma}}{\left(\frac{L_{1}}{L}\right)^{\gamma}-\left(\frac{L_{1}}{L}\right)^{-\gamma}}\left(\frac{L}{x}\right)^{\frac{\beta}{\sigma}}
$$

From (4.2), Proposition 5 is obtained.

Note that when $L_{1}$ approaches infinity (the barrier becomes less and less likely to be reached), then the ratio in (4.2) converges to $\left(\frac{L}{x}\right)^{\xi}$ given in (4.1).

In the case of a down-and-out put, the value can be written the same way but it is simplified; exercise is optimal just before the barrier, unless the barrier is beyond the optimum level. Hence,

$$
\begin{aligned}
P_{\mathrm{A}}^{\mathrm{do}}(x) & =\sup _{L} \mathbb{E}_{x}\left[\mathrm{e}^{-r T_{L \vee L_{1}}}\left(K-L \vee L_{1}\right)\right] \\
& =\frac{K}{\xi+1}\left(\frac{K \xi}{x(\xi+1)}\right)^{\xi} \mathbb{I}_{L_{1}<\frac{\xi}{\xi+1} K}+\left(K-L_{1}\right)\left(\frac{L_{1}}{x}\right)^{\xi} \mathbb{I}_{L_{1} \geq \frac{\xi}{\xi+1}} K^{\cdot}
\end{aligned}
$$

\subsection{The perpetual American Parisian put}

In this section we derive closed-form expressions for the value of the perpetual American Parisian options, given a constant exercise frontier. Unfortunately the complexity of the expressions prevents us from finding a closed form expression for the exercise frontier itself; we can only write the equation it should solve. We use the results shown in Appendix B regarding the Laplace transforms of $H_{D}^{+} \wedge T_{a}$ and $H_{D}^{-} \wedge T_{a}$. These results generalize the analysis of the Parisian stopping time from [5]. The Parisian case is more complex than the simple barriers because at the Parisian time the underlying price can be anywhere above the barrier (or below, for a "down" option).

\section{The Parisian up-and-out put}

As mentioned in Sect. 3 for the call, the exercise boundary is constant.

Proposition 6 The value of the perpetual American Parisian up-and-out put option with the barrier at $L_{1}$ verifies 


$$
\begin{aligned}
P_{\mathrm{AP}}^{\mathrm{uo}}(x) & =\sup _{L} \mathbb{E}_{x}\left[\mathrm{e}^{-r T_{L}}(K-L) \mathbb{I}_{T_{L}<H_{L_{1}, D}^{+}}\right] \\
& =\sup _{L}\left\{(K-L)\left(\frac{L}{x}\right)^{\frac{\beta}{\sigma}}\left(\frac{\left(\frac{L_{1}}{x}\right)^{\gamma}-\left(\frac{L_{1}}{x}\right)^{-\gamma}}{\left(\frac{L_{1}}{L}\right)^{\gamma}-\left(\frac{L_{1}}{L}\right)^{-\gamma}}+\frac{\mathrm{E}}{\mathrm{F}} \frac{\left(\frac{L}{x}\right)^{\gamma}-\left(\frac{L}{x}\right)^{-\gamma}}{\left(\frac{L}{L_{1}}\right)^{\gamma}-\left(\frac{L}{L_{1}}\right)^{-\gamma}}\right)\right\},
\end{aligned}
$$

with

$$
\begin{aligned}
\mathrm{E}= & 2 \sqrt{\pi D \rho} \mathrm{e}^{\rho D}\left(\left(\frac{L_{1}}{L}\right)^{-\gamma}-\Phi(-\sqrt{2 \rho D})\right)+\left(\left(\frac{L_{1}}{L}\right)^{\gamma}-\left(\frac{L_{1}}{L}\right)^{-\gamma}\right) \\
& \times(\Phi(\sqrt{2 \rho D})-\Phi(-\sqrt{2 \rho D})), \\
\mathrm{F}= & 2 \sqrt{\pi D \rho} \mathrm{e}^{\rho D}\left(1-\Phi(-\sqrt{2 \rho D})\left(\frac{L_{1}}{L}\right)^{-\gamma}\right) \\
& +\Phi(\sqrt{2 \rho D})\left(\left(\frac{L_{1}}{L}\right)^{\gamma}-\left(\frac{L_{1}}{L}\right)^{-\gamma}\right)\left(\left(\frac{L_{1}}{L}\right)^{-\gamma}-\Phi(-\sqrt{2 \rho D})\right)
\end{aligned}
$$

and with $\gamma=\frac{1}{\sigma} \sqrt{2 \rho}, \rho=r+\frac{\beta^{2}}{2}, L_{1} \geq K$ and the function $\Phi$ given in Proposition 2.

Let us note the decomposition of this value as a function of the non-Parisian option. For a given exercise frontier $L$ (but not necessarily at the optimum), the value of the Parisian option reads

$$
\begin{aligned}
\mathbb{E}_{S_{0}}\left[\mathrm{e}^{-r T_{L}}(K-L) \mathbb{I}_{T_{L}<H_{L_{1}, D}^{+}}\right]= & \mathbb{E}_{S_{0}}\left[\mathrm{e}^{-r T_{L}}(K-L) \mathbb{I}_{T_{L}<T_{L_{1}}}\right] \\
& +\mathbb{E}_{S_{0}}\left[\mathrm{e}^{-r T_{L}}(K-L) \mathbb{I}_{T_{L_{1}} \leq T_{L}<H_{L_{1}, D}^{+}}\right]
\end{aligned}
$$

For a given exercise policy, the Parisian option is worth at least the value of the simple barrier option, plus a term that depends on what happens once the barrier has been hit. Note that here $\mathbb{E}_{S_{0}}\left[\mathrm{e}^{-r T_{L}}(K-L) \mathbb{I}_{T_{L}<T_{L_{1}}}\right]$ is not equal to $P_{\mathrm{A}}^{\mathrm{uo}}(x)$ since the optimal exercise will not necessarily be the same in the Parisian and non-Parisian cases.

Proof of Proposition 6 To obtain this option's value, we need to calculate $\mathbb{E}_{x}\left[\mathrm{e}^{-r T_{L}}(K-L) \mathbb{I}_{T_{L}<H_{L_{1}, D}^{+}}\right]$. In fact, we rather calculate the purely Parisian term $\mathbb{E}_{x}\left[\mathrm{e}^{-r T_{L}}(K-L) \mathbb{I}_{T_{L_{1}} \leq T_{L}<H_{L_{1}, D}^{+}}\right]$and obtain the incremental value of the Parisian option over the non-Parisian option, for a given exercise frontier.We 
have, by applying the strong Markov property of Brownian motion at $T_{L_{1}}$, that

$$
\begin{aligned}
\mathbb{E}_{x}\left[\mathrm{e}^{-r T_{L}}(K-L) \mathbb{I}_{T_{L_{1}} \leq T_{L}<H_{L_{1}, D}^{+}}\right]= & \mathbb{E}_{x}\left[\mathrm{e}^{\left.-r T_{L_{1}} \mathbb{I}_{T_{L_{1}} \leq T_{L}}\right]}\right. \\
& \times \mathbb{E}_{L_{1}}\left[\mathrm{e}^{-r T_{L}}(K-L) \mathbb{I}_{T_{L}<H_{L_{1}, D}^{+}}\right]
\end{aligned}
$$

Using Girsanov's theorem at time $T_{L}$, we obtain

$$
\mathbb{E}_{L_{1}}\left[\mathrm{e}^{-r T_{L}}(K-L) \mathbb{I}_{T_{L}<H_{L_{1}, D}^{+}}\right]=(K-L)\left(\frac{L}{x}\right)^{\frac{\beta}{\sigma}} \mathbb{E}\left[\mathrm{e}^{-\left(r+\frac{\beta^{2}}{2}\right) T_{l}} \mathbb{I}_{T_{l}<H_{D}^{+}}\right]
$$

where $H_{D}^{+}$stands for $H_{0, D}^{+}$. We know that the last factor in (4.3) can be calculated; Theorem 1 in Appendix B gives the desired result. Note that the theorem is actually given for $H_{D}^{-}$and $T_{a}$, where $a$ is positive. Here, we are in the opposite situation, so we simply need to apply the theorem to the Brownian motion $-W$ instead of $W$. We have, with $l=\frac{1}{\sigma} \ln \frac{L}{x}$ and $\rho=r+\frac{\beta^{2}}{2}$,

$$
\begin{aligned}
& \mathbb{E}\left[\mathrm{e}^{-\rho T_{l}} \mathbb{I}_{T_{l}<H_{D}^{+}}\right]=\frac{1}{\mathrm{e}^{l \sqrt{2 \rho}}-\Phi(-\sqrt{2 \rho D})} \\
& \quad \times\left(1-\Phi(-\sqrt{2 \rho D}) \frac{\sqrt{\pi D \rho} \mathrm{e}^{\rho D}+\sinh (l \sqrt{2 \rho})}{\sqrt{\pi D \rho} \mathrm{e}^{\rho D-l \sqrt{2 \rho}}+\sinh (l \sqrt{2 \rho}) \Phi(\sqrt{2 \rho D})}\right) .
\end{aligned}
$$

After a few simplifications, we obtain $\mathbb{E}\left[\mathrm{e}^{-\rho T_{l}} \mathbb{I}_{T_{l}<H_{D}^{+}}\right]=\frac{\mathrm{E}}{\mathrm{F}}$ with $\mathrm{E}$ and $\mathrm{F}$ defined in the proposition. Now, putting the pieces back together we have

$$
\begin{aligned}
\mathbb{E}_{x}\left[\mathrm{e}^{-r T_{L}}(K-L) \mathbb{I}_{T_{L_{1}} \leq T_{L}<H_{L_{1}, D}^{+}}\right]= & \frac{\mathrm{E}}{\mathrm{F}} \frac{\left(\frac{L}{x}\right)^{\gamma}-\left(\frac{L}{x}\right)^{-\gamma}}{\left(\frac{L}{L_{1}}\right)^{\gamma}-\left(\frac{L}{L_{1}}\right)^{-\gamma}} \\
& \times(K-L)\left(\frac{L_{1}}{x}\right)^{\frac{\beta}{\sigma}}\left(\frac{L}{L_{1}}\right)^{\frac{\beta}{\sigma}} .
\end{aligned}
$$

\section{The Parisian down-and-out put}

Because of the asymmetry of the up-and-down Parisian options, the downand-out is very different from the up-and-out. As for up-and-out barrier calls (see Sect. 3), we cannot write a closed-form expression for this option. As with the non-Parisian case, if the barrier is lower than the optimal entry level in the non-Parisian, non-barrier case, then the results are trivial. However, if the barrier is higher, the optimal exercise frontier is complicated (see the equivalent case of the up-and-out Parisian call). As a simplification of the exercise frontier, we propose to use the first instant where either the underlying reaches 
the Parisian time or hits a certain constant level lower than the barrier. Exercise takes place at the optimal level or right before the Parisian time. This way, the simplified exercise frontier is "square", being hit either as a function of time, or as a function of the level. With this simplification regarding the exercise frontier, we have the following

Proposition 7 The value of the perpetual down-and-out American Parisian put option is approximated as follows

$$
\begin{aligned}
P_{\mathrm{AP}}^{\mathrm{do}}(x) & \approx \sup _{L} \mathbb{E}_{x}\left[\mathrm { e } ^ { - r H _ { L _ { 1 } , D ^ { \wedge } } ^ { - } } \left(K-T_{L}\right.\right. \\
& =\sup _{L}\left(\frac{L_{1}}{x}\right)^{\sqrt{2 \sigma^{-2} \rho} D^{\wedge}} \frac{\left[(1-\mathrm{AB})(K-L)+\left(\mathrm{e}^{a \sqrt{2 \rho}} \mathrm{B}-1\right) \mathrm{K}(f)\right]}{\mathrm{e}^{a \sqrt{2 \rho}}-\mathrm{A}}
\end{aligned}
$$

for $L_{1} \leqslant K$, with $\rho=r+\frac{\beta^{2}}{2}, a=\frac{1}{\sigma} \ln \frac{L}{L_{1}}, f(z)=\mathrm{e}^{\beta z}\left(K-L_{1} \mathrm{e}^{\sigma z}\right)$, and where $\mathrm{A}$, $\mathrm{B}$ and $\mathrm{K}(f)$ are defined at the end of Appendix $B$.

The latter decomposition is different from the one in the up-and-out case. The first part is the value attached to exercising when the process has reached the optimal level, while the second is the value of exercising right before the Parisian time, $D$ units of time after the excursion below $L_{1}$ started.

If the exercise price is below the Parisian threshold $L_{1}$, it is not always optimal to exercise the option at the Parisian time. In that case, if the Parisian time happens before the optimal exercise barrier is hit, then depending on whether the underlying is below the strike price, the option is either exercised or not.

Proof The proof is a direct application of the main result (Theorem 1) from [14].

\section{Conclusion}

In this article, the problem of American Parisian option pricing is tackled. A probabilistic approach is proposed. Just as for the standard American option context, pricing formulas depend on the exercise boundary. In the perpetual case, exercise boundaries and therefore prices can be derived or approximated.

\section{Appendix A: Simplifying the perpetual case}

The first integral on the right-hand side of the second decomposition given in Proposition 1 can be rewritten as follows (see the Subsect. 2.1). We have 


$$
\begin{aligned}
\int_{0}^{+\infty} C_{\mathrm{EP}}^{\mathrm{do}}\left(x, L_{C}, u\right) \mathrm{d} u= & \int_{0}^{+\infty} \exp \left(-\left(r+\frac{\beta^{2}}{2}\right) u\right) * C_{E}\left(x, L_{C}, u\right) \mathrm{d} u \\
& -\int_{0}^{+\infty} \exp \left(-\left(r+\frac{\beta^{2}}{2}\right) u\right) * C_{\mathrm{EP}}^{\mathrm{di}}\left(x, L_{C}, u\right) \mathrm{d} u,
\end{aligned}
$$

where $C_{E}\left(x, L_{C}, u\right)$ is the price of the standard European currency option (given by [12]) with strike price $L_{C}$ and maturity $u$. Moreover,

$$
\begin{aligned}
{ }^{*} C_{E}\left(x, L_{C}, u\right) & =\exp \left(\left(r+\frac{\beta^{2}}{2}\right) u\right) C_{E}\left(x, L_{C}, u\right) \\
& =\mathbb{E}\left[\left(x \exp \left(\sigma Z_{u}\right)-L_{C}\right)^{+} \exp \left(\beta Z_{u}\right)\right]
\end{aligned}
$$

and

$$
\begin{aligned}
{ }^{*} C_{\mathrm{EP}}^{\mathrm{di}}\left(x, L_{C}, u\right) & =\exp \left(\left(r+\frac{\beta^{2}}{2}\right) u\right) C_{\mathrm{EP}}^{\mathrm{di}}\left(x, L_{C}, u\right) \\
& =\mathbb{E}\left[\mathbb{I}_{H_{l_{1}, D}^{-}(Z) \leqslant u}\left(x \exp \left(\sigma Z_{u}\right)-L_{C}\right)^{+} \exp \left(\beta Z_{u}\right)\right] .
\end{aligned}
$$

All the expectations in Appendix A, including those on the right-hand sides of (A.2) and (A.3), are computed by relying on the probability $\mathbb{P}$ under which $\left(Z_{t}=W_{t}+\beta t, t \geqslant 0\right)$, is a Brownian motion.

Let us first consider the second integral on the right-hand side of (A.1). From [5],

$$
\begin{aligned}
& \int_{0}^{+\infty} \exp \left(-\left(r+\frac{\beta^{2}}{2}\right) u\right) * C_{\mathrm{EP}}^{\mathrm{di}}\left(x, L_{C}, u\right) \mathrm{d} u \\
& =\int_{0}^{+\infty} \mathrm{d} u \exp \left(-\left(r+\frac{\beta^{2}}{2}\right) u\right) \int_{-\infty}^{+\infty} \mathrm{d} y f_{x}(y) h_{l_{1}}(u, y),
\end{aligned}
$$

where $\beta$ and $l_{1}$ are defined in Proposition 1 ,

$$
f_{x}(y)=\exp (\beta y)\left(x \exp (\sigma y)-L_{C}\right)^{+}
$$

and

$$
h_{l_{1}}(u, y)=\int_{-\infty}^{+\infty} \mathbb{E}\left[\mathbb{I}_{H_{l_{1}, D}^{-}(Z) \leqslant u} \frac{\exp \left(-\frac{\left(l_{1}-z-y\right)^{2}}{2\left(u-H_{l_{1}, D}^{-}(Z)\right)}\right)}{\sqrt{2 \pi\left(u-H_{l_{1}, D}^{-}(Z)\right)}}\right] v(\mathrm{~d} z),
$$


and where $v(\mathrm{~d} z)$ denotes the law of $Z_{H_{l_{1}, D}^{-}(Z)}$ from (3.12). Hence,

$$
\begin{aligned}
& \int_{0}^{+\infty} \exp \left(-\left(r+\frac{\beta^{2}}{2}\right) u\right) * C_{\mathrm{EP}}^{\mathrm{di}}\left(x, L_{C}, u\right) \mathrm{d} u \\
& \quad=\int_{l_{C}}^{+\infty} \mathrm{d} y f_{x}(y) \int_{0}^{+\infty} \mathrm{d} u \exp \left(-\left(r+\frac{\beta^{2}}{2}\right) u\right) h_{l_{1}}(u, y),
\end{aligned}
$$

where $l_{C}=\ln \left(L_{C} / x\right) / \sigma$. The Laplace transform of $h_{l_{1}}(., y)$ is known (see [5]). Indeed, if $L_{C}>L_{1}$ (this is the case because $L_{C}>K \geqslant L_{1}$ ), we have

$$
\begin{aligned}
& \int_{0}^{+\infty} \exp \left(-\left(r+\frac{\beta^{2}}{2}\right) u\right) h_{l_{1}}(u, y) \mathrm{d} u \\
& \quad=\frac{\Phi\left(-\sqrt{\left(2 r+\beta^{2}\right) D}\right)}{\Phi\left(\sqrt{\left(2 r+\beta^{2}\right) D}\right)} \frac{\exp \left(\left(2 l_{1}-y\right) \sqrt{2 r+\beta^{2}}\right)}{\sqrt{2 r+\beta^{2}}} \quad \text { for } y>l_{C},
\end{aligned}
$$

where the function $\Phi$ is given in Proposition 2. Therefore by definition of the function $f_{x}$ given in (A.4),

$$
\begin{aligned}
& \int_{0}^{+\infty} \exp \left(-\left(r+\frac{\beta^{2}}{2}\right) u\right) * C_{\mathrm{EP}}^{\mathrm{di}}\left(x, L_{C}, u\right) \mathrm{d} u \\
& =\frac{\Phi\left(-\sqrt{\left(2 r+\beta^{2}\right) D}\right)}{\Phi\left(\sqrt{\left(2 r+\beta^{2}\right) D}\right)} \frac{\exp \left(2 l_{1} \sqrt{2 r+\beta^{2}}\right)}{\sqrt{2 r+\beta^{2}}} \\
& \quad \times\left(\frac{x}{\sqrt{2 r+\beta^{2}}-\beta-\sigma}\left(\frac{x}{L_{C}}\right)^{\frac{\sqrt{2 r+\beta^{2}}-\beta-\sigma}{\sigma}}-\frac{L_{C}}{\sqrt{2 r+\beta^{2}}-\beta}\left(\frac{x}{L_{C}}\right)^{\frac{\sqrt{2 r+\beta^{2}}-\beta}{\sigma}}\right) .
\end{aligned}
$$

Indeed it is straightforward to show that $\beta+\sigma-\sqrt{2 r+\beta^{2}}<0, \forall \delta>0$.

The Laplace transform of ${ }^{*} C_{E}\left(x, L_{C}, u\right)$ can be obtained by relying on (A.7) with $D=l_{1}=0$. Hence, (A.1) can be rewritten as

$$
\begin{aligned}
& \int_{0}^{+\infty} C_{\mathrm{EP}}^{\mathrm{do}}\left(x, L_{C}, u\right) \mathrm{d} u=\left(\frac{x}{L_{C}}\right)^{\frac{\sqrt{2 r+\beta^{2}}-\beta}{\sigma}} L_{C}\left(\frac{1}{\sqrt{2 r+\beta^{2}}-\beta-\sigma}-\frac{1}{\sqrt{2 r+\beta^{2}}-\beta}\right) \\
& \quad \times\left(1-\frac{\Phi\left(-\sqrt{\left(2 r+\beta^{2}\right) D}\right)}{\Phi\left(\sqrt{\left(2 r+\beta^{2}\right) D}\right)} \exp \left(2 l_{1} \sqrt{2 r+\beta^{2}}\right)\right) \frac{1}{\sqrt{2 r+\beta^{2}}} .
\end{aligned}
$$

Concerning the second integral of the decomposition given in Proposition 2, we have 


$$
\begin{aligned}
& \int_{0}^{+\infty} \operatorname{BINC}_{\mathrm{EP}}^{\mathrm{do}}\left(x, L_{C}, u\right) \mathrm{d} u=-\frac{\partial\left(\int_{0}^{+\infty} C_{\mathrm{EP}}^{\mathrm{do}}\left(x, L_{C}, u\right) \mathrm{d} u\right)}{\partial L_{C}} \\
& =\left(\frac{\sqrt{2 r+\beta^{2}}-\beta}{\sigma}-1\right)\left(\frac{1}{\sqrt{2 r+\beta^{2}}-\beta-\sigma}-\frac{1}{\sqrt{2 r+\beta^{2}}-\beta}\right) \\
& \quad \times\left(\frac{x}{L_{C}}\right)^{\frac{\sqrt{2 r+\beta^{2}}-\beta}{\sigma}}\left(1-\frac{\Phi\left(-\sqrt{\left(2 r+\beta^{2}\right) D}\right)}{\Phi\left(\sqrt{\left(2 r+\beta^{2}\right) D}\right)} \exp \left(2 l_{1} \sqrt{2 r+\beta^{2}}\right)\right) \frac{1}{\sqrt{2 r+\beta^{2}}} .
\end{aligned}
$$

Finally, by relying on (A.7) and (A.9), the first decomposition of Proposition 2 can be rewritten as

$$
\begin{aligned}
C_{\mathrm{AP}}^{\mathrm{do}}(x)= & \left(1-\frac{\Phi\left(-\sqrt{\left(2 r+\beta^{2}\right) D}\right)}{\Phi\left(\sqrt{\left(2 r+\beta^{2}\right) D}\right)} \exp \left(2 l_{1} \sqrt{2 r+\beta^{2}}\right)\right) \frac{1}{\sqrt{2 r+\beta^{2}}} \\
& \times\left(\frac{1}{\sqrt{2 r+\beta^{2}}-\beta-\sigma}-\frac{1}{\sqrt{2 r+\beta^{2}}-\beta}\right)\left(\frac{x}{L_{C}}\right) \\
& \times \delta\left(L_{C}+\left(L_{C}-\frac{r}{\delta} K\right)\left(\frac{\sqrt{2 r+\beta^{2}}-\beta}{\sigma}-\beta\right.\right. \\
\sigma & 1)) .
\end{aligned}
$$

By relying on the definition of $\theta$, the option value given in Proposition 2 is derived. By setting $x=L_{C}$ in this option value, the last result of Proposition 2 is obtained.

Using again the definition of $\theta$, this result in the case of the standard American option $\left(L_{1} \rightarrow 0\right)$ reads

$$
L_{C}-K=\frac{1}{\sigma(\theta \sigma+\beta)}\left(\frac{\delta L_{C}}{\theta-1}-\frac{r}{\theta} K\right) .
$$

Hence, by the definition of $\beta$ and by relying on the fact that $\theta$ is the positive root of the equation $\frac{\sigma^{2}}{2} y^{2}+\beta \sigma y-r=0$, the well-known result $L_{C}=\frac{\theta}{\theta-1} K$ is obtained.

\section{Appendix B: Passage times and excursions}

The study of the perpetual case for American Parisian options boils down to the study of the stopping times $H_{D}^{+} \wedge T_{a}$ and $H_{D}^{-} \wedge T_{a}$ with $a>0$. In the first case, this is the first instant when a positive excursion reaches $a$ or lasts for more than $D$. In the second case, it is the first instant when a negative excursion lasts more than $D$ or a positive excursion reaches $a$. Intuitively, the second case should be 
simpler than the first one, since we can separate the positive excursions from the negative ones.

We shall see that, indeed, the resulting formulas are much less cumbersome. The study of some of the characteristics of $H_{D}^{+} \wedge T_{a}$ was carried out in [14]. To perform the same analysis on $H_{D}^{-} \wedge T_{a}$, we have to adapt the approach developed in that paper. In fact, the calculation is slightly simpler in this case. We consider in detail the problem raised by the down-and-out call, or similarly the up-and-out put, that involves $H_{D}^{-} \wedge T_{a}$.

The Down-and-out call or the Up-and-out put: $H_{D}^{-} \wedge T_{a}$

We follow a similar approach to that in the proof given in [14]. We first give our result.

Theorem 1 We have the following relationships for a standard Brownian motion $B$, a positive number $\rho$, a positive level $a$, and a measurable function bounded below $f$ :

$$
\begin{aligned}
\mathbb{E}\left[\mathrm{e}^{-\rho T_{a}} \mathbb{I}_{H_{D}^{-} \geq T_{a}}\right] & =\frac{1-\Phi(-\sqrt{2 \rho D}) \mathrm{X}}{\mathrm{e}^{a \sqrt{2 \rho}}-\Phi(-\sqrt{2 \rho D})}, \\
\mathbb{E}\left[\mathrm{e}^{-\rho H_{D}^{-}} \mathbb{I}_{H_{D}^{-} \leq T_{a}} f\left(W_{H_{D}^{-}}\right)\right] & =\frac{1-\Phi(-\alpha \sqrt{D}) \mathrm{X}}{\mathrm{e}^{\alpha a}-\Phi(-\alpha \sqrt{D})} \int_{0}^{+\infty} f(-\alpha z \sqrt{D}) z \mathrm{e}^{-\frac{z^{2}}{2}} \mathrm{~d} z
\end{aligned}
$$

with $\Phi(y)=1+\sqrt{2 \pi} y \mathrm{e}^{\frac{y^{2}}{2}} \mathcal{N}(y) \quad$ and $\quad \mathrm{X}=\frac{\sqrt{\pi D \rho} \mathrm{e}^{\rho D}+\sinh (a \sqrt{2 \rho})}{\sqrt{\pi D \rho} \mathrm{e}^{\rho D-a \sqrt{2 \rho}}+\sinh (a \sqrt{2 \rho}) \Phi(\sqrt{2 \rho D})}$.

To show this result, we use excursion theory and some of the results of [5].

Lemma 1 For all $\alpha$, we have

$$
\mathbb{E}\left[\mathrm{e}^{-\frac{\alpha^{2}}{2} T_{a}} \mathbb{I}_{H_{D}^{-} \geq T_{a}}\right]=\frac{1-\Phi(-\alpha \sqrt{D}) \mathbb{E}\left[\mathrm{e}^{-\frac{\alpha^{2}}{2} H_{D}^{-} \wedge T_{a}}\right]}{\mathrm{e}^{\alpha a}-\Phi(-\alpha \sqrt{D})} .
$$

Proof Studying such a problem it is natural to try and use exponential martingale properties. First we write

$$
\mathbb{E}\left[\mathrm{e}^{-\rho H_{D}^{-} \wedge T_{a}}\right]=\mathbb{E}\left[\mathrm{e}^{-\rho T_{a}} \mathbb{I}_{H_{D}^{-} \geq T_{a}}\right]+\mathbb{E}\left[\mathrm{e}^{-\rho H_{D}^{-}} \mathbb{I}_{H_{D}^{-} \leq T_{a}}\right] .
$$

Let us now define the exponential martingale $\left(M_{t}=\exp \left(\alpha W_{t}-\frac{\alpha^{2}}{2} t\right), t \geq 0\right)$, for any real $\alpha$. If we apply Doob's martingale stopping theorem, we obtain $1=\mathbb{E}\left[\mathrm{e}^{\alpha W_{H_{D^{-}} \wedge T_{a}}-\frac{\alpha^{2}}{2} H_{D}^{-} \wedge T_{a}}\right]=\mathbb{E}\left[\mathrm{e}^{\alpha W_{H_{D}^{-}}-\frac{\alpha^{2}}{2} H_{D}^{-}} \mathbb{I}_{H_{D}^{-} \leq T_{a}}\right]+\mathrm{e}^{\alpha a} \mathbb{E}\left[\mathrm{e}^{-\frac{\alpha^{2}}{2} T_{a}} \mathbb{I}_{H_{D}^{-} \geq T_{a}}\right]$. 
We know that $H_{D}^{-}$and $\left(W_{t}\right)$ for $g\left(H_{D}^{-}\right) \leq t \leq H_{D}^{-}$are independent, and as a consequence, $W_{H_{D}^{-}}$and $\sup _{g\left(H_{D}^{-}\right) \leq t \leq H_{D}^{-}} W_{t}$ are independent of $H_{D}^{-}$. So, we can write

$$
\mathbb{E}\left[\mathrm{e}^{\alpha W_{H_{D}^{-}-\frac{\alpha^{2}}{2} H_{D}^{-}}} \mathbb{I}_{H_{D}^{-} \leq T_{a}}\right]=\mathbb{E}\left[\mathrm{e}^{\alpha W_{H_{D}^{-}}}\right] \mathbb{E}\left[\mathrm{e}^{-\frac{\alpha^{2}}{2} H_{D}^{-}} \mathbb{I}_{H_{D}^{-} \leq T_{a}}\right]
$$

However, we know that the trajectory $\left(W_{t}, g\left(H_{D}^{-}\right) \leq t \leq H_{D}^{-}\right)$is a Brownian meander $m$, and by scaling

$$
\left(W_{t}, g\left(H_{D}^{-}\right) \leq t \leq H_{D}^{-}\right)=\left(-\sqrt{D} m_{u}, 0 \leq u \leq 1\right) \text { in law. }
$$

Consequently, $\mathbb{E}\left[\mathrm{e}^{\alpha W_{H_{D}^{-}}}\right]=\mathbb{E}\left[\mathrm{e}^{-\alpha \sqrt{D} m_{1}}\right]=\Phi(-\alpha \sqrt{D})$. Gathering the results, we have

$$
1=\Phi(-\alpha \sqrt{D}) \mathbb{E}\left[\mathrm{e}^{-\frac{\alpha^{2}}{2} H_{D}^{-}} \mathbb{I}_{H_{D}^{-} \leq T_{a}}\right]+\mathrm{e}^{\alpha a} \mathbb{E}\left[\mathrm{e}^{-\frac{\alpha^{2}}{2} T_{a}} \mathbb{I}_{H_{D}^{-} \geq T_{a}}\right] .
$$

This gives a second relationship between the quantities we are interested in. When combining this with (B.1), we get the result stated in the lemma.

In the preceding argument, we use the fact that $W_{H_{D}^{-}}$is independent from $H_{D}^{-}$and $\mathbb{I}_{H_{D}^{-} \leq T_{a}}$. We can use this to write

$$
\mathbb{E}\left[\mathrm{e}^{-\rho H_{D}^{-}} \mathbb{I}_{H_{D}^{-} \leq T_{a}} f\left(W_{H_{D}^{-}}\right)\right]=\mathbb{E}\left[\mathrm{e}^{-\rho H_{D}^{-}} \mathbb{I}_{H_{D}^{-} \leq T_{a}}\right] \int_{0}^{+\infty} f(-\alpha z \sqrt{D}) z \mathrm{e}^{-\frac{z^{2}}{2}} \mathrm{~d} z .
$$

Besides, the term $\mathbb{E}\left[\mathrm{e}^{-\rho H_{D}^{-}} \mathbb{I}_{H_{D}^{-} \leq T_{a}}\right]$ can be calculated by using (B.2) and (B.1). We obtain

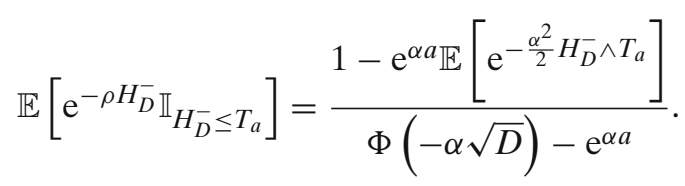

To show the theorem, we simply have to calculate $\mathbb{E}\left[\mathrm{e}^{-\rho H_{D}^{-} \wedge T_{a}}\right]$.

Lemma 2 For all positive $\rho$, we have

$\mathbb{E}\left[\mathrm{e}^{-\rho H_{D}^{-} \wedge T_{a}}\right]=1-\rho \frac{\int \mathbf{n}(\mathrm{d} \varepsilon) \int_{0}^{V(\varepsilon)} \mathrm{d} v \mathrm{e}^{-\rho v}\left(\mathbb{I}_{\varepsilon_{v}>0} \mathbb{S}_{\text {sup }_{u \leq v} \varepsilon_{u} \leq a}+\mathbb{I}_{\varepsilon_{v} \leq 0} \mathbb{I}_{v<D}\right)}{\int \mathbf{n}(\mathrm{d} \varepsilon)\left(1-\mathrm{e}^{-\rho V(\varepsilon)}\left(\mathbb{I}_{\varepsilon \leq 0} \mathbb{I}_{V(\varepsilon)<D}+\mathbb{I}_{\varepsilon>0} \mathbb{I}_{\text {sup }_{0 \leq u \leq V(\varepsilon)} \varepsilon_{u}<a}\right)\right)}$

where $\mathbf{n}$ is Itô's excursion measure. 
Proof The stopping time we are interested in is $\tau=H_{D}^{-} \wedge T_{a}$, and we write

$$
\mathbb{E}\left[\int_{0}^{\infty} \mathrm{e}^{-\rho t} \mathbb{I}_{\tau \geq t} \mathrm{~d} t\right]=\frac{1}{\rho}\left(1-\mathbb{E}\left[\mathrm{e}^{-\rho \tau}\right]\right)
$$

so

$$
\mathbb{E}\left[\mathrm{e}^{-\rho \tau}\right]=1-\rho \mathbb{E}\left[\int_{0}^{\infty} \mathrm{e}^{-\rho t} \mathbb{I}_{\tau \geq t} \mathrm{~d} t\right]
$$

We define $\tau_{s}$ as the inverse of the local time process (the notation $\tau_{s}$ is conventional for this process); in other words, $\tau_{s}=\inf \left\{u: L_{u}=s\right\}$ increases only when the Brownian motion is at zero. We also define the longest negative excursion up to a time $\tau_{s-}$ as

$$
l^{-}\left(\tau_{s-}\right)=\sup \left\{l \geq 0: \exists u \leq s,\left(\tau_{u}-\tau_{u-}\right)=l, \mathbf{e}_{u} \leq 0\right\},
$$

and we introduce the highest level reached by an excursion up to time $\tau_{s-}$ defined by

$$
h^{+}\left(\tau_{s-}\right)=\sup \left\{h \geq 0: \exists u \leq s, \sup _{\tau_{u-} \leq v \leq \tau_{u}} \mathbf{e}_{u}(v)=l, \mathbf{e}_{u} \geq 0\right\},
$$

where $\mathbf{e}$ is the excursion process (see for example [20] for a description of the excursion process). These random variables can also be defined up to the last zero as

$$
\begin{aligned}
l^{-}\left(g_{t}\right) & =\sup \left\{\left(d_{s}-g_{s}\right): g_{t} \geq s \geq 0, W_{s} \leq 0\right\}, \\
h^{+}\left(g_{t}\right) & =\sup \left\{W_{s}: g_{t} \geq s \geq 0, W_{s} \geq 0\right\}
\end{aligned}
$$

$V$ will be used to denote the length of an excursion, that is, $V\left(\mathbf{e}_{s}\right)=\tau_{s}-\tau_{s-}$.

Now, let us note that we have the following equalities of events:

$$
\begin{aligned}
(\tau>t)= & \left\{D>l^{-}\left(g_{t}\right)\right\} \cap\left(\left\{W_{t} \geq 0\right\} \cup\left(\left\{W_{t}<0\right\} \cap\left\{t-g_{t}<D\right\}\right)\right) \\
& \cap\left\{h^{+}\left(g_{t}\right)<a\right\} \cap\left(\left\{W_{t} \leq 0\right\} \cup\left(\left\{W_{t}>0\right\} \cap\left\{\sup _{g_{t} \leq u \leq t} W_{u} \leq a\right\}\right)\right) .
\end{aligned}
$$

The equation above states simply that $\tau>t$ is equivalent to saying that "the longest negative excursion, up to the last zero before $t$, is shorter than $D$, and the highest excursion up to that time is smaller than $a$ " and "since $g_{t}$, either the Brownian motion went up but its maximum has not reached $a$, or it went down and $D$ units of time have not yet passed." So, 


$$
\begin{aligned}
\int_{0}^{\infty} \mathbb{E}\left[\mathrm{e}^{-\rho t} \mathbb{I}_{\tau \leq t}\right] \mathrm{d} t=\mathbb{E} \int_{0}^{\infty} \mathrm{e}^{-\rho t} \mathbb{I}_{l^{-}\left(g_{t}\right)<D}\left(\mathbb{I}_{W_{t}<0} \mathbb{I}_{t-g_{t}<D}+\mathbb{I}_{W_{t} \geq 0}\right) \\
\quad \times \mathbb{I}_{h^{+}\left(g_{t}\right)<a}\left(\mathbb{I}_{W_{t}>0} \mathbb{I}_{\sup _{g_{t} \leq u \leq t} W_{u} \leq a}+\mathbb{I}_{W_{t} \leq 0}\right) \mathrm{d} t .
\end{aligned}
$$

Let us recall the following balayage theory result, which can be found for example in [22]. For two functionals $F_{1}$ and $F_{2}$,

$$
\begin{aligned}
& \mathbb{E}\left[\int_{0}^{\infty} \mathrm{e}^{-\rho t} F_{1}\left(B_{u}, u \leq g_{t}\right) F_{2}\left(B_{u}, g_{t} \leq u \leq t\right) \mathrm{d} t\right] \\
& =\mathbb{E}\left[\int_{0}^{\infty} \mathrm{e}^{-\rho \tau_{s-}} F_{1}\left(B_{u}, u \leq \tau_{s-}\right) \mathrm{d} s\right] \int \mathbf{n}(\mathrm{d} \varepsilon) \int_{0}^{V(\varepsilon)} \mathrm{d} v \mathrm{e}^{-\rho v} F_{2}\left(\varepsilon_{u}, u \leq v\right) .
\end{aligned}
$$

Applying the result to the problem at hand, we get

$$
\begin{aligned}
\mathbb{E} \int_{0}^{\infty} \mathrm{e}^{-\rho t} \mathbb{I}_{l^{-}\left(g_{t}\right)<D}\left(\mathbb{I}_{W_{t}<0} \mathbb{I}_{t-g_{t}<D}+\mathbb{I}_{W_{t} \geq 0}\right) \mathbb{I}_{h^{+}\left(g_{t}\right)<a}\left(\mathbb{I}_{W_{t}>0} \mathbb{I}_{\sup _{g_{t} \leq u \leq t} W_{u} \leq a}+\mathbb{I}_{W_{t} \leq 0}\right) \mathrm{d} t \\
=\mathbb{E}\left[\int_{0}^{\infty} \mathrm{e}^{-\rho \tau_{s}} \mathbb{I}_{l^{-}\left(\tau_{s}\right)<D} \mathbb{I}_{h^{+}\left(\tau_{s}\right)<a} \mathrm{~d} s\right] \\
\quad \times \int \mathbf{n}(\mathrm{d} \varepsilon) \int_{0}^{V(\varepsilon)} \mathrm{d} v \mathrm{e}^{-\rho v}\left(\mathbb{I}_{\mathcal{E}_{v}<0} \mathbb{I}_{v<D}+\mathbb{I}_{\mathcal{E}_{v} \geq 0}\right)\left(\mathbb{I}_{\mathcal{E}_{v}>0} \mathbb{I}_{\sup _{u \leq v} \varepsilon_{u} \leq a}+\mathbb{I}_{\mathcal{E}_{v} \leq 0}\right) .
\end{aligned}
$$

We now turn to the computation of the path integral in the expression above.

We are interested in $\mathbb{E}\left[\int_{0}^{\infty} \mathrm{e}^{-\rho \tau_{s}} \mathbb{I}_{l^{-}\left(\tau_{s}\right)<D} \mathbb{I}_{h^{+}\left(\tau_{s}\right)<a} \mathrm{~d} s\right]$. We start with

$$
\begin{aligned}
& \mathrm{e}^{-\rho \tau_{s}} \mathbb{I}_{l^{-}\left(\tau_{s}\right)<D} \mathbb{I}_{h^{+}\left(\tau_{s}\right)<a} \\
& \quad=1+\sum_{0 \leq u \leq s}\left(\mathrm{e}^{-\rho \tau_{u}} \mathbb{I}_{l^{-}\left(\tau_{u}\right)<D} \mathbb{I}_{h^{+}\left(\tau_{u}\right)<a}-\mathrm{e}^{-\rho \tau_{u^{-}}} \mathbb{I}_{l^{-}\left(\tau_{u^{-}}\right)<D} \mathbb{I}_{h^{+}\left(\tau_{u^{-}}\right)<a}\right) .
\end{aligned}
$$

But we also easily obtain that

$$
\begin{aligned}
& \left\{l^{-}\left(\tau_{u}\right)<D\right\} \cap\left\{h^{+}\left(\tau_{u}\right)<a\right\} \\
& =\left\{l^{-}\left(\tau_{u-}\right)<D\right\} \cap\left(\left\{\mathbf{e}_{u} \geq 0\right\} \cup\left(\left\{\mathbf{e}_{u}<0\right\} \cap\left\{\tau_{u}-\tau_{u-}<D\right\}\right)\right) \\
& \quad \cap\left\{h^{+}\left(\tau_{u-}\right)<a\right\} \cap\left(\left\{\mathbf{e}_{u} \leq 0\right\} \cup\left(\left\{\mathbf{e}_{u}>0\right\} \cap\left\{\sup _{\tau_{u-} \leq v \leq \tau_{u}} \mathbf{e}_{u}(v)<a\right\}\right)\right) .
\end{aligned}
$$


Hence,

$$
\begin{aligned}
& \mathrm{e}^{-\rho \tau_{s}} \mathbb{I}_{l^{-}\left(\tau_{s}\right)<D} \mathbb{I}_{h^{+}\left(\tau_{s}\right)<a} \\
& =1+\sum_{0 \leq u \leq s} \mathrm{e}^{-\rho \tau_{u-}} \mathbb{I}_{l^{-}\left(\tau_{u-}\right)<D} \mathbb{I}_{h^{+}\left(\tau_{u-}\right)<a} \\
& \quad \times\left(\mathrm{e}^{-\rho V\left(\mathbf{e}_{s}\right)}\left(\mathbb{I}_{\mathbf{e}_{u} \geq 0}+\mathbb{I}_{\mathbf{e}_{u}<0} \mathbb{I}_{\tau_{u}-\tau_{u-}<D}\right)\left(\mathbb{I}_{\mathbf{e}_{u} \leq 0}+\mathbb{I}_{\mathbf{e}_{u}>0} \mathbb{I}_{\sup _{\tau_{u-} \leq v \leq \tau_{u}}} \mathbf{e}_{u}(v)<a\right)-1\right) .
\end{aligned}
$$

Taking the expectation and applying the compensation formula yields

$$
\begin{aligned}
\mathbb{E} & {\left[\mathrm{e}^{-\rho \tau_{s}} \mathbb{I}_{l^{-}\left(\tau_{s}\right)<D} \mathbb{I}_{h^{+}\left(\tau_{s}\right)<a}\right] } \\
= & 1+\mathbb{E}\left[\int_{0}^{s} \mathrm{e}^{-\rho \tau_{u-}} \mathbb{I}_{l^{-}\left(\tau_{s}\right)<D} \mathbb{I}_{h^{+}\left(\tau_{s}\right)<a} \mathrm{~d} u\right] \\
& \times \int \mathbf{n}(\mathrm{d} \varepsilon)\left(\mathrm{e}^{-\rho V(\varepsilon)}\left(\mathbb{I}_{\varepsilon \geq 0}+\mathbb{I}_{\varepsilon<0} \mathbb{I}_{V(\varepsilon)<D}\right)\left(\mathbb{I}_{\varepsilon \leq 0}+\mathbb{I}_{\varepsilon>0} \mathbb{I}_{\sup _{0 \leq u \leq V(\varepsilon)} \varepsilon_{u}<a}\right)-1\right) .
\end{aligned}
$$

If we define $\varphi_{D}(s)=\mathbb{E}\left[\mathrm{e}^{-\rho \tau_{s}} \mathbb{I}_{l^{-}\left(\tau_{s}\right)<D} \mathbb{I}_{h^{+}\left(\tau_{s}\right)<a}\right]$, then we have $\mathbb{E}\left[\int_{0}^{\infty} \mathrm{e}^{-\rho \tau_{s}} \mathbb{I}_{l^{-}\left(\tau_{s}\right)<D} \mathbb{I}_{h^{+}\left(\tau_{s}\right)<a} \mathrm{~d} s\right]=\int_{0}^{\infty} \varphi_{D}(s) \mathrm{d} s$, and

$$
\begin{aligned}
& \varphi_{D}(s)=1+\int \mathbf{n}(\mathrm{d} \varepsilon) \\
& \quad\left(\mathrm{e}^{-\rho V(\varepsilon)}\left(\mathbb{I}_{\varepsilon \geq 0}+\mathbb{I}_{\varepsilon<0} \mathbb{I}_{V(\varepsilon)<D}\right)\left(\mathbb{I}_{\varepsilon \leq 0}+\mathbb{I}_{\varepsilon>0} \mathbb{I}_{\sup _{0 \leq u \leq V(\varepsilon)} \varepsilon_{u}<a}\right)-1\right) \int_{0}^{s} \varphi_{D}(u) \mathrm{d} u .
\end{aligned}
$$

Solving the differential equation and rewriting the entire expression directly gives the result stated in Lemma 2.

We now turn to the actual calculation of these integrals. We summarize the result as follows.

Lemma 3 For all positive $\rho$,

$$
\mathbb{E}\left[\mathrm{e}^{-\rho \tau}\right]=\frac{\sqrt{\pi D \rho} \mathrm{e}^{\rho D}+\sinh (a \sqrt{2 \rho})}{\sqrt{\pi D \rho} \mathrm{e}^{\rho D-a \sqrt{2 \rho}}+\sinh (a \sqrt{2 \rho})\left(1+2 \sqrt{\pi D \rho} \mathrm{e}^{\rho D \mathcal{N}(\sqrt{2 \rho D}))} .\right.}
$$

Proof To compute these integrals, we have to use the "law" under Itô's measure of the supremum and the length of an excursion. We use Williams' and Itô's description of $\mathbf{n}$. Williams' description states that $\mathbf{n}\left(\sup _{u \leq V(\varepsilon)} \varepsilon_{u} \in \mathrm{d} m\right)=\frac{\mathrm{d} m}{m^{2}}$, and that the excursion path conditionally on $m$ is composed of two Bessel-3 processes put back to back between 0 and their hitting times of $m$. Itô's description of the measure $\mathbf{n}$ is based on the excursion's length and says that $\mathbf{n}(V(\varepsilon) \in \mathrm{d} v)=\frac{\mathrm{d} v}{\sqrt{2 \pi v^{3}}}$. Conditional on this length, the excursion path is a 
Bessel-3 bridge. In fact, we simply use Itô's law of the excursion length. First, let us see how these laws intervene in our problem. For the denominator,

$$
\begin{aligned}
& \int \mathbf{n}(\mathrm{d} \varepsilon)\left(1-\mathrm{e}^{-\rho V(\varepsilon)}\left(\mathbb{I}_{\varepsilon \leq 0} \mathbb{I}_{V(\varepsilon)<D}+\mathbb{I}_{\varepsilon>0} \mathbb{I}_{\sup _{0 \leq u \leq V(\varepsilon)} \varepsilon_{u}<a}\right)\right) \\
& =\int \mathbf{n}(\mathrm{d} \varepsilon) \mathbb{I}_{\varepsilon \leq 0}\left(1-\mathrm{e}^{-\rho V(\varepsilon)} \mathbb{I}_{V(\varepsilon)<D}\right) \\
& \quad+\int \mathbf{n}(\mathrm{d} \varepsilon) \mathbb{I}_{\varepsilon>0}\left(1-\mathrm{e}^{-\rho V(\varepsilon)} \mathbb{I}_{\sup _{0 \leq u \leq V(\varepsilon)} \varepsilon_{u}<a}\right)
\end{aligned}
$$

and for the numerator,

$$
\begin{aligned}
& \int \mathbf{n}(\mathrm{d} \varepsilon) \int_{0}^{V(\varepsilon)} \mathrm{d} v \mathrm{e}^{-\rho v}\left(\mathbb{I}_{\varepsilon_{v}>0} \mathbb{I}_{\sup _{u \leq v} \varepsilon_{u} \leq a}+\mathbb{I}_{\mathcal{E}_{v} \leq 0} \mathbb{I}_{v<D}\right) \\
& =\int \mathbf{n}(\mathrm{d} \varepsilon) \int_{0}^{V(\varepsilon)} \mathrm{d} v \mathrm{e}^{-\rho v} \mathbb{I}_{\varepsilon_{v}>0} \mathbb{I}_{\sup _{u \leq v} \varepsilon_{u} \leq a}+\int \mathbf{n}(\mathrm{d} \varepsilon) \int_{0}^{V(\varepsilon)} \mathrm{d} v \mathrm{e}^{-\rho v} \mathbb{I}_{v<D} \mathbb{I}_{\varepsilon_{v} \leq 0} .
\end{aligned}
$$

Note that restricting the integrals over positive or negative excursions just divides them by 2 . Since these restrictions appear in all expressions, both in the numerator and denominator, we simplify them. We have

$$
\begin{aligned}
\int \mathbf{n}(\mathrm{d} \varepsilon) \int_{0}^{V(\varepsilon)} \mathrm{d} v \mathrm{e}^{-\rho v} \mathbb{I}_{\sup _{u \leq v} \varepsilon_{u} \leq a} & =\int \mathbf{n}(\mathrm{d} \varepsilon) \int_{0}^{V(\varepsilon)} \mathrm{d} v \mathrm{e}^{-\rho v} \mathbb{I}_{T_{a}(\varepsilon)<v} \\
& =\frac{1}{\rho} \int \mathbf{n}(\mathrm{d} \varepsilon)\left(1-\mathrm{e}^{-\rho\left(V(\varepsilon) \wedge T_{a}(\varepsilon)\right)}\right)
\end{aligned}
$$

and, following exactly the same reasoning,

$$
\begin{aligned}
\int \mathbf{n}(\mathrm{d} \varepsilon) \int_{0}^{V(\varepsilon)} \mathrm{d} v \mathrm{e}^{-\rho v} \mathbb{I}_{v<D} & =\int \mathbf{n}(\mathrm{d} \varepsilon) \int_{0}^{V(\varepsilon) \wedge D} \mathrm{~d} v \mathrm{e}^{-\rho v} \\
& =\frac{1}{\rho} \int \mathbf{n}(\mathrm{d} \varepsilon)\left(1-\mathrm{e}^{-\rho(V(\varepsilon) \wedge D)}\right) .
\end{aligned}
$$

Therefore, (B.4) (multiplied by 2) can be rewritten as

$$
\frac{1}{\rho} \int \mathbf{n}(\mathrm{d} \varepsilon)\left(1-\mathrm{e}^{-\rho\left(V(\varepsilon) \wedge T_{a}(\varepsilon)\right)}\right)+\frac{1}{\rho} \int \mathbf{n}(\mathrm{d} \varepsilon)\left(1-\mathrm{e}^{-\rho(V(\varepsilon) \wedge D)}\right) .
$$

These expressions can be calculated using Williams' description of Itô's measure. Let us note that, conditional on the maximum of the excursion $m$, the law 
of the life length $V$ is the sum of two independent hitting times $T_{m}(R)$ for a Bessel(3) process. We have

$$
T_{a}(\varepsilon) \wedge V(\varepsilon)=\left(T_{m}(R)+T_{m}\left(R^{\prime}\right)\right) \mathbb{I}_{m<a}+T_{a}(R) \mathbb{I}_{m \geq a} \quad \text { in law }
$$

for an independent Bessel(3) process $R^{\prime}$. From [4, p. 339, formula 2.0.1] we get $\mathbb{E}_{a}\left[\mathrm{e}^{-\frac{\alpha^{2}}{2} T_{h}(R)}\right]$ for a Bessel(3) process $R$ starting from $a$, with $h \geq a$. Taking the limit as $a$ goes to zero, we have

$$
\mathbb{E}_{0}\left[\mathrm{e}^{-\frac{\alpha^{2}}{2} T_{h}(R)}\right]=\frac{\alpha h}{\sinh (\alpha h)}
$$

We obtain for (B.3) (times 2) the expression

$$
\begin{aligned}
& \int \mathbf{n}(\mathrm{d} \varepsilon)\left(1-\mathrm{e}^{-\rho V(\varepsilon)} \mathbb{I} V(\varepsilon)<D\right)+\int \mathbf{n}(\mathrm{d} \varepsilon)\left(1-\mathrm{e}^{-\rho V(\varepsilon)} \mathbb{I}_{\sup _{0 \leq u \leq V(\varepsilon)} \varepsilon_{u}<a}\right) \\
& =\frac{2}{\sqrt{2 \pi D}}+\int_{0}^{D} \frac{\mathrm{d} v}{\sqrt{2 \pi v^{3}}}\left(1-\mathrm{e}^{-\rho v}\right)+\frac{1}{a}+\int_{0}^{a} \frac{\mathrm{d} m}{m^{2}}\left(1-\mathbb{E}_{0}^{(3)}\left[\mathrm{e}^{-\rho\left(T_{m}+T_{m}^{\prime}\right)}\right]\right) .
\end{aligned}
$$

However, $\mathbb{E}_{0}^{(3)}\left[\mathrm{e}^{-\rho\left(T_{m}+T_{m}^{\prime}\right)}\right]=\left(\mathbb{E}_{0}^{(3)}\left[\mathrm{e}^{-\rho T_{2 m}(R)}\right]\right)^{2}=\frac{2 \rho m^{2}}{\sinh ^{2}(m \sqrt{2 \rho})}$, so

$$
\int_{0}^{a} \frac{\mathrm{d} m}{m^{2}}\left(1-\mathbb{E}_{0}^{(3)}\left[\mathrm{e}^{-\rho\left(T_{m}+T_{m}^{\prime}\right)}\right]\right)=\sqrt{2 \rho}-\frac{1}{a}-\frac{4 \rho}{\sqrt{2 \rho}\left(1-\mathrm{e}^{2 a \sqrt{2 \rho}}\right)}
$$

Consequently, (B.5) can be written as

$$
\frac{2}{\sqrt{2 \pi D}}+\int_{0}^{D} \frac{\mathrm{d} v}{\sqrt{2 \pi v^{3}}}\left(1-\mathrm{e}^{-\rho v}\right)+\sqrt{2 \rho}-\frac{4 \rho}{\sqrt{2 \rho}\left(1-\mathrm{e}^{2 a \sqrt{2 \rho}}\right)} .
$$

Let us now turn to the numerator. We have (using B.6)

$$
\begin{gathered}
\frac{1}{\rho} \int \mathbf{n}(\mathrm{d} \varepsilon)\left(1-\mathrm{e}^{-\rho\left(V(\varepsilon) \wedge T_{a}(\varepsilon)\right)}\right)+\frac{1}{\rho} \int \mathbf{n}(\mathrm{d} \varepsilon)\left(1-\mathrm{e}^{-\rho(V(\varepsilon) \wedge D)}\right) \\
=\frac{1}{\rho}\left(\sqrt{2 \rho}-\frac{4 \rho}{\sqrt{2 \rho}\left(1-\mathrm{e}^{2 a \sqrt{2 \rho}}\right)}\right)-\frac{1}{\rho} \int_{a}^{\infty} \frac{\mathrm{d} m}{m^{2}} \frac{a \sqrt{2 \rho}}{\sinh (a \sqrt{2 \rho})} \\
+\frac{1}{\rho} \int_{0}^{D} \frac{\mathrm{d} v}{\sqrt{2 \pi v^{3}}}\left(1-\mathrm{e}^{-\rho v}\right)+\frac{\left(1-\mathrm{e}^{-\rho D}\right)}{\rho} \int_{D}^{\infty} \frac{\mathrm{d} v}{\sqrt{2 \pi v^{3}}} .
\end{gathered}
$$


Now, we can gather all the terms and the result stated in Lemma 3 is obtained.

Combining our results, Theorem 1 is finally proved.

Based on Lemma 3, we can quickly see that $\tau$ behaves like $T_{a}$ when $D$ is very large, and like $H_{D}^{-}$when $a$ is large, in agreement with the result of [5].

The up-and-out call or the down-and-out put: $H_{D}^{+} \wedge T_{a}$

In the case of the up-and-out call or down-and-out put [14] gives a closed form expression for $\mathbb{E}\left[\mathrm{e}^{-\rho T_{a}} \mathbb{I}_{H_{D}^{+} \geq T_{a}}\right], \quad \mathbb{E}\left[\mathrm{e}^{-\rho H_{D}^{+}} \mathbb{I}_{H_{D}^{+} \leq T_{a}} f\left(W_{H_{D}^{+}}\right)\right], \quad$ and $\mathbb{E}\left[\mathrm{e}^{-\rho T_{a} \wedge H_{D}^{+} f}\left(W_{T_{a} \wedge H_{D}^{+}}\right)\right]$. In the result, the following quantities are used:

$$
\begin{aligned}
& \mathrm{K}(f)=\sum_{k \in \mathbb{Z}} \int_{0}^{\frac{a}{\sqrt{D}}} \mathrm{~d} z f(z \sqrt{D})\left(z+\frac{2 k a}{\sqrt{D}}\right) \exp \left(-\frac{1}{2}\left(z+\frac{2 k a}{\sqrt{D}}\right)^{2}\right), \\
& \mathrm{A}=\frac{\sum_{k \in \mathbb{Z}} \int_{0}^{\frac{a}{\sqrt{D}}} \mathrm{~d} z\left(z+\frac{2 k a}{\sqrt{D}}\right) \exp \left(\sqrt{2 \rho D} z-\frac{1}{2}\left(z+\frac{2 k a}{\sqrt{D}}\right)^{2}\right)}{2 \sum_{k \in \mathbb{Z}} \exp \left(-\frac{2 k^{2} a^{2}}{D}\right)\left(1-\exp \left(-\frac{(4 k+1) a^{2}}{2 D}\right)\right)}, \\
& \mathrm{B}=1-\frac{\int_{0}^{a} \frac{\mathrm{d} m}{m^{2}}\left(1-\mathbb{E}_{0}^{(3)}\left[\mathrm{e}^{-\rho\left(T_{m}+T_{m}^{\prime}\right) \wedge D}\right]\right)}{\int_{0}^{a} \frac{\mathrm{d} m}{m^{2}}\left(1-\mathbb{E}_{0}^{(3)}\left[\mathrm{e}^{-\rho\left(T_{m}+T_{m}^{\prime}\right)} \mathbb{I}_{T_{m}+T_{m}^{\prime}<D}\right]\right)+\frac{1}{a}+\sqrt{2 \rho}} \\
& -\frac{\int_{0}^{\infty} \frac{\mathrm{d} v}{\sqrt{2 \pi v^{3}}}\left(1-\mathrm{e}^{-\rho v}\right)+\frac{1}{a}\left(1-\mathbb{E}_{0}^{(3)}\left[\mathrm{e}^{-\rho T_{a} \wedge D}\right]\right)}{\int_{0}^{a} \frac{\mathrm{d} m}{m^{2}}\left(1-\mathbb{E}_{0}^{(3)}\left[\mathrm{e}^{-\rho\left(T_{m}+T_{m}^{\prime}\right)} \mathbb{I}_{T_{m}+T_{m}^{\prime}<D}\right]\right)+\frac{1}{a}+\sqrt{2 \rho}} .
\end{aligned}
$$

Acknowledgments This research was supported by the University Research Priority Program "Finance and Financial Markets" and by the National Center of Competence in Research FINRISK, research instruments of the University of Zurich and of the Swiss National Science Foundation, respectively. This paper has also benefited from the helpful comments of Monique Jeanblanc and Ganna Reshetar.

\section{References}

1. Avellaneda, M., Wu, L.: Pricing Parisian-style options with a lattice method. IJTAF 2, 1-16 (1999)

2. Ben Ameur, H., L'Ecuyer, P., Lemieux, C.: Variance reduction of Monte Carlo and randomized quasi Monte Carlo estimators for stochastic volatility models in finance. In: Proceedings of the 1999 Winter Simulation Conference, pp. 336-343. IEEE Press (1999)

3. Bernard, C., Le Courtois, O., Quittard Pinon, F.: A new procedure for pricing Parisian options. J. Deriv. 12, 45-54 (2005)

4. Borodin, A., Salminen, P.: Handbook of Brownian Motion-Facts and Formulae. Birkhäuser (1996) 
5. Chesney, M., Jeanblanc, M., Yor, M.: Brownian excursions and Parisian barrier options. Adv. Appl. Probab. 29, 165-184 (1997)

6. Cornwall, M.J., Chesney, M., Jeanblanc-Picqué, M., Kentwell, G.W., Yor, M.: Parisian barrier options: a discussion. Risk Mag. 10,77-79 (1997)

7. Costabile, M.: A combinatorial approach for pricing Parisian options. Decis. Econ. Financ. 25, 111-125 (2002)

8. Detemple, J.: American options symmetry properties. In: Jouini, E., Cvitanić, J., Musiela, M. (eds.), Option Pricing, Interest Rates and Risk Management, pp. 67-104. Cambridge University Press, Cambridge (2001)

9. El Karoui N., Jeanblanc, M., Bellamy, N.: Options exotiques. Finance 20, $49-67$ (1999)

10. Fujita, T., Miura, R.: Edokko options: a new framework of barrier options. Asia-Pac. Financ. Mark. 9, 141-151 (2002)

11. Gao, B., Huang, J., Subramahnyam, M.: The valuation of American barrier options using the decomposition technique. J. Econ. Dyn. Control 24, 1783-1827 (2000)

12. Garman, M.B., Kohlhagen, S.W.: Foreign currency option values. J. Int. Money Financ. 2, 231237 (1983)

13. Gauthier, L.: Options réelles et options exotiques, une approche probabiliste. Thèse de Mathématiques, Université Paris 1 Panthéon-Sorbonne (2000)

14. Gauthier, L.: Excursions height- and length-related stopping times, and application to finance. Adv. Appl. Probab. 34, 846-868 (2002)

15. Grau, A.J.: Moving windows. Working Paper. University of Waterloo, Canada (2003)

16. Grau, A.J., Kallsen, J.: Speedy Monte Carlo pricing of path-dependent options. Working Paper, Technische Universität München, Germany (2004)

17. Haber, R., Schönbucher, P., Wilmott, P.: Pricing Parisian options. J. Deriv. 6, 71-79 (1999)

18. McDonald, R.L., Schroder, M.: A parity result for American options. J. Comput. Financ. 1, 5-13 (1998)

19. Mordecki, E.: Optimal stopping for a diffusion with jumps. Financ. Stoch. 3, 227-236 (1999)

20. Revuz, D., Yor, M.: Continuous Martingales and Brownian motion, 3rd edn. Springer, Berlin Heidelberg New York (1999)

21. Wilmott, P.: Derivatives. Wiley, New York (1998)

22. Yor, M.: Local times and excursion for Brownian motion: a concise introduction. Lecciones in Matematicas, Universidad Central de Venezuela (1997) 